\title{
Identification of Substituted Amino Acid Hydrazides as Novel Anti-Tubercular Agents, Using a Scaffold Hopping Approach
}

\author{
Alistair K. Brown ${ }^{1}{ }^{\complement}$, Ahmed K. B. Aljohani ${ }^{1}$, Fatimah M. A. Alsalem ${ }^{1}$, Joseph L. Broadhead ${ }^{2}$, \\ Jason H. Gill ${ }^{3,4}\left(\mathbb{D}\right.$, Yucheng $\mathrm{Lu}^{1}$ and Jonathan D. Sellars ${ }^{1,4, *(\mathbb{D})}$ \\ 1 Biosciences Institute, Faculty of Medical Sciences, Newcastle University, Newcastle upon Tyne NE2 4HH, \\ UK; alistair.brown2@newcastle.ac.uk (A.K.B.); a.aljohani2@newcastle.ac.uk (A.K.B.A.); \\ f.m.a.alsalem2@newcastle.ac.uk (F.M.A.A.); y.lu48@newcastle.ac.uk (Y.L.) \\ 2 Arcinova, Taylor Drive, Alnwick NE66 2DH, UK; joseph190597@googlemail.com \\ 3 Translational and Clinical Research Institute, Faculty of Medical Sciences, Newcastle University, \\ Newcastle upon Tyne NE2 4HH, UK; jason.gill@newcastle.ac.uk \\ 4 School of Pharmacy, Faculty of Medical Sciences, King George VI Building, \\ Newcastle upon Tyne NE1 7RU, UK \\ * Correspondence: jon.sellars@newcastle.ac.uk; Tel.: +44-191-2082357
}

Academic Editor: Derek J. McPhee

Received: 22 April 2020; Accepted: 15 May 2020; Published: 21 May 2020

\begin{abstract}
Discovery and development of new therapeutic options for the treatment of Mycobacterium tuberculosis $(M t b)$ infection, particularly drug-resistant strains, are urgently required to tackle the global burden of this disease. Herein, we reported the synthesis of a novel series of $\mathrm{N}$-substituted amino acid hydrazides, utilising a scaffold hopping approach within a library of anti-tubercular agents. Efficacy and selectivity were evaluated against three strains of $M t b$ (wild-type, isoniazid-resistant and rifampicin-resistant), and cytotoxicity against macrophages in vitro. The antibacterial activity and therapeutic index of these molecules were significantly affected by modifications with the $\mathrm{N}$-substituents. Introduction of a 3,5-dinitroaryl moiety demonstrated enhanced antibacterial activity against all three strains of $M t b$. In contrast, the inclusion of an imidazo [1,2-a]pyridine-3-carboxy moiety resulted in enhanced activity towards isoniazid mono-resistant $M t b$ relative to wild-type $M t b$. Consequently, this scaffold hopping approach showed significant promise for exemplification of novel molecules with specific activity profiles against drug-resistant tuberculosis.
\end{abstract}

Keywords: antibacterial; Mycobacterium tuberculosis; amino acid; hydrazide; Q203; Naphthalene; Quinoline; scaffold hopping

\section{Introduction}

The World Health OrganisatioN's (WHO) global tuberculosis report in 2019 recognises tuberculosis (TB) as a communicable disease responsible for a major cause of long term illness and continues to be one of the top ten causes of death worldwide from a single infectious agent [1]. In 2018, an estimated ten million (range 9.0-11.1 million) new cases of TB infection were reported, equivalent to 132 cases per 100,000 population globally [2]. Unfortunately, treatment responses to TB are stagnating, and most WHO regions are now likely to fail to reach the 2020 milestone of the WHO's End TB strategy [2]. Concerningly, there is an ever-increasing number of infections from strains resistant to the front line anti-TB drugs-rifampicin (RIF) and isoniazid (INH), exacerbating further the global TB epidemic [1]. Consequently, new drug molecules with novel modes of action are urgently required to address this increased disease burden and diminishing response to current therapies. 
Over the past ten years, notable advances towards the development of new therapies against TB have been made. For instance, the dihydro-nitroimidazooxazole derivative-Delamanid (Deltyba ${ }^{\circledR}$ ) —acts as a prodrug activated by the mycobacterial deazaflavin-dependent nitroreductase to an inhibitor of methoxy mycolic acid and ketomycolic acid synthesis of the mycobacterial cell wall [3]. Another such drug, dinitrobenzamide (DNB-1) has shown a novel mechanism involving inhibition of decaprenylphosphoryl- $\beta$-d-ribose 2 '-epimerase (DprE1), a vital enzyme in cell wall lipoarabinomannan and arabinogalactan precursor biosynthesis [4]. Conversely, pyrazinamide, a clinically utilised drug since 1952 demonstrates activity against dormant or semi-dormant TB, with a mode of action involving multiple cellular targets focusing on the disruption of energy production and fatty acid synthesis [5]. However, despite the significant promise, resistance to several of these molecules has now been reported [5-9].

A notable new direction for the development of anti-TB drugs has been the perturbation of the mycobacterial respiratory electron transport chain (ETC), with several such drugs entering the clinic recently (Figure 1) [10]. The diarylquinoline Bedaquiline (SIRTURO $\left.{ }^{\circledR}{ }^{\circledR}\right)$, used predominantly against drug-resistant TB, targets the mycobacterial respiratory chain enzyme F1/F0-ATPase [11,12]. Whereas the 1,2-ethylene diamine SQ109, shown to be active against both drug-sensitive and drug-resistant TB, has a mechanism involving inhibition of the Mycobacterial membrane protein Large 3 (MmpL3)-dependent export of mycolic acids but has also been linked to the dissipation of the proton motive force within the ETC [13]. More recently, the first in class drug-Q203 (Telacebec ${ }^{\circledR \circledR}$ ), has shown a novel mechanism of action targeting the cytochrome $b c 1$ complex of the ETC and has demonstrated significant promise in the clinic [14]. Furthermore, the therapeutic mechanism of the 'old' drug clofazimine, which has been repurposed for treatment of drug-resistant $\mathrm{TB}$, also inhibits the NADH dehydrogenase (NAD-2) within the ETC [15]. Consequently, in order to tackle the problematic exacerbation of drug-resistant TB, the identification of drug targets outside of the conventional pathways for current drugs and subsequent discovery of drug molecules exploiting these new therapeutic avenues are required.

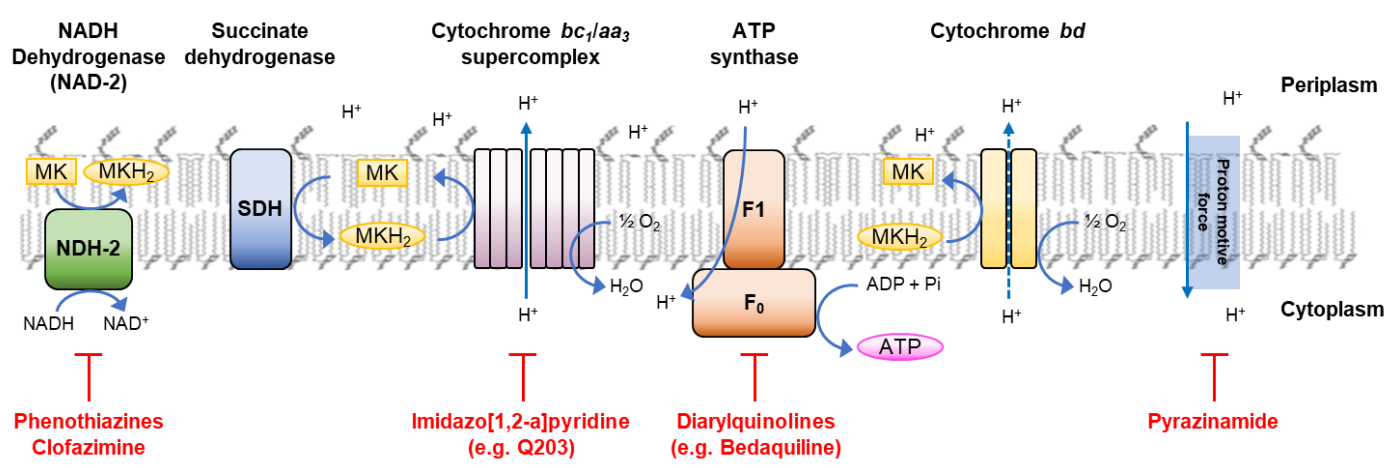

Figure 1. Respiratory electron transport chain (ETC) in Mycobacterium tuberculosis (Mtb). Electrons derived from NADH are fed into the electron transport chain by the type II NADH dehydrogenase (NDH-2), leading to the reduction of the menaquinone pool $\left(\mathrm{MK} / \mathrm{MKH}_{2}\right)$. The menaquinone pool can also be reduced by alternative electron donors, e.g., the succinate dehydrogenase (SDH). The menaquinone pool transfers electrons to the cytochrome bc1 complex. The cytochrome bc1 complex forms a supercomplex with the cytochrome aa3-type terminal oxidase, which transfers the electrons onto oxygen. Additionally, oxygen can be reduced by the cytochrome bd-type terminal oxidase, which directly accepts electrons from the menaquinone pool. Electron transport along the ETC promotes proton motive force; this energy is utilised by ATP synthase, resulting in the synthesis of ATP.

Our previous studies of benzoxa-[2,1,3]-diazole-substituted amino acid hydrazides demonstrated therapeutic selectivity of these molecules against mycolata over Gram-positive and Gram-negative bacteria [16]. This highlighted the involvement of the benzoxa-[2,1,3]-diazole, the amino acid $\left(\mathrm{R}_{2}\right)$ moiety and the substituted aryl hydrazine $\left(\mathrm{R}_{1}\right)$ towards bacterial selectivity, efficacy and establishment 
of a therapeutic index for development as a drug [16]. In this study, we evaluated variation of the benzoxa-[2,1,3]-diazole moiety within these compounds via the introduction of new architectures and their structure-activity-relationships toward the development of efficacious drugs against drug-resistant $M t b$ (Figure 2).
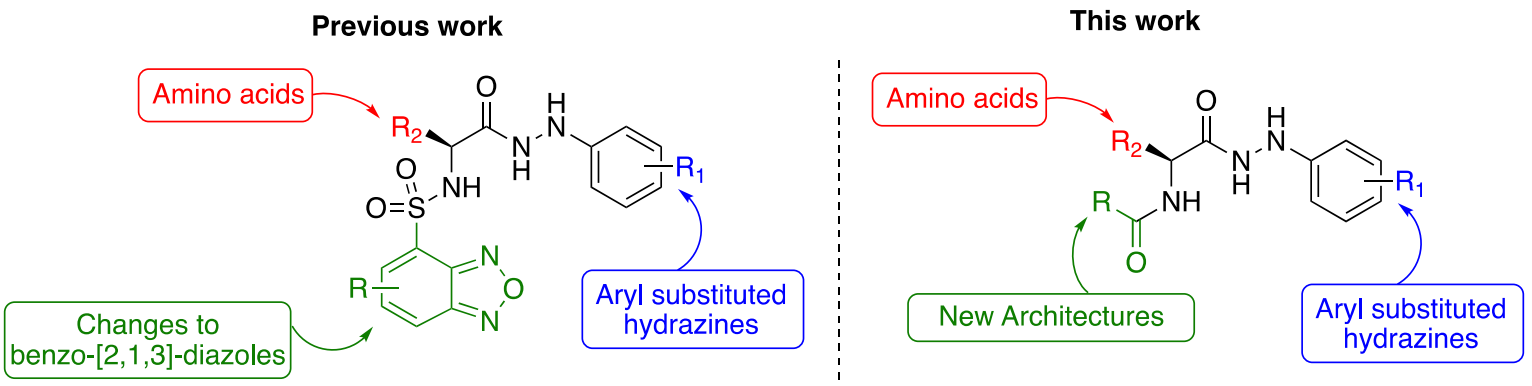

Figure 2. Inhibitors of M. tuberculosis based on a benzoxa-[2,1,3]-diazole framework, highlighting the key modifications.

\section{Strategy}

Previously, we established the importance of the amino acid and hydrazide moieties within benzoxa-[2,1,3]-diazole-substituted amino acid hydrazides for selective therapeutic activity against $M t b$ [16]. The current study expanded upon this previous work and evaluated the impact of specific alteration of the benzoxa-[2,1,3]-diazole moiety towards the development of therapeutics with improved anti-M $M b$ activity, particularly towards drug-resistant $M t b$. To achieve this objective, we adopted the scaffold hopping approach of known pharmacophores to identify putative molecules [17-19]. This predominantly focused on the utilisation of pharmacophores with known anti-Mtb activity amenable to incorporation at the site of the current benzoxa-[2,1,3]-diazole moiety. We specifically evaluated pharmacophores from drugs targeting the mycobacterial cell wall, isoniazid (INH) $\mathbf{1}$ and the 3,5-dinitrobenzene moiety of DNB-1, 2; the multi-target drug Pyrazinamide 3; the new generation of molecules perturbing the mycobacterial ETC, the quinoline and naphthalene of Bedaquiline 4 and the imidazo [1,2-a]pyridine-3-carboxy moiety of Q203 5 (Figure 3).

Both the amino acid and hydrazide moieties were locked within the structure, utilising 4-trifluoromethylphenyl 6-7 and 3-chlorophenyl hydrazine 8-9. In terms of the amino acids, we previously demonstrated alanine (Ala) to exhibit the highest activity when conjugated within these hydrazides [16]. To recapitulate the sulphur moiety present within several known anti-TB drugs [20], amino acids with this functionality were previously evaluated within the hydrazides. As cysteine was nucleophilic and not amenable to the introduction, methionine (Met) was utilised. Linkage of Met to either the 4-trifluoromethylphenyl or the 3-chlorophenyl hydrazine produced comparable therapeutic activity against $M t b$ and a therapeutic index similar to that of $\mathbf{6}$, containing Ala. The choice of hydrazides was based on the fact that 4-trifluoromethylphenyl hydrazide 6 had exhibited moderate activity against $M t b$ and a significant therapeutic index relative to cytotoxicity against mammalian cells, and 3-chlorophenyl hydrazine 8 had demonstrated notable impact upon $M t b$ therapeutic activity [16].

The objective of this study was to evaluate the importance of modifying the benzoxa-[2,1,3]-diazole moiety scaffold with those from other molecules with known anti-TB activity in order to appraise the therapeutic impact of substituents at this position and the potential for exploitation towards the development of molecules with demonstrated activity against drug-resistant $M t b$. 
<smiles>NNC(=O)c1ccncc1</smiles>

1<smiles>NC(=O)c1cnccn1</smiles>

3<smiles>COc1ccc(OCCNC(=O)c2cc([N+](=O)[O-])cc([N+](=O)[O-])c2)cc1</smiles>

2<smiles>COc1nc2ccc(Br)cc2cc1[C@@H](c1ccccc1)[C@@](O)(CCN(C)C)c1cccc2ccccc12</smiles>

4<smiles>CCc1nc2ccc(Cl)cn2c1C(=O)NCc1ccc(N2CCC(c3ccc(OC(F)(F)F)cc3)CC2)cc1</smiles>

5

Figure 3. Scaffold hopping architectures (green) of molecules with known anti-tuberculosis (TB) activity evaluated within this study.

\section{Results and Discussion}

\subsection{Chemical Synthesis of Substituted Amino Acid Hydrazides}

Previously established methods were employed to synthesise the substituted amino acid hydrazides starting from $N$-Boc amino acids and the corresponding hydrazide (Scheme 1).

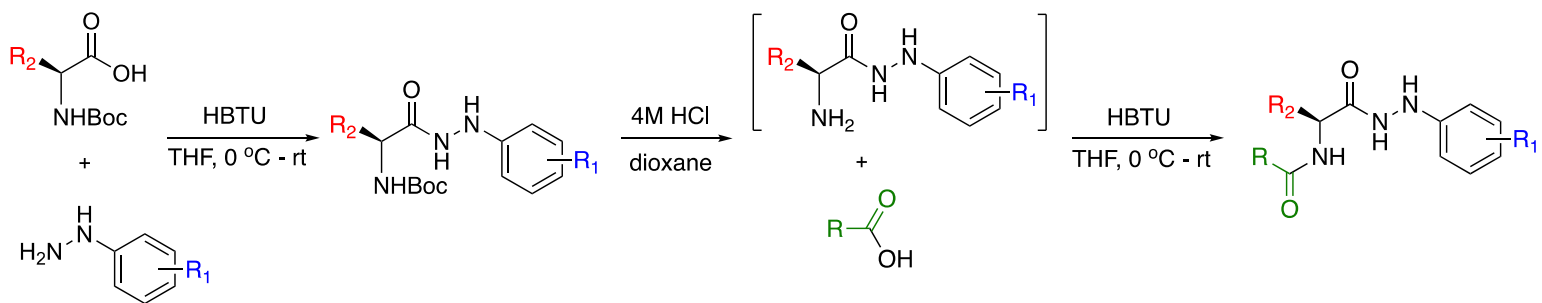

Scheme 1. Synthesis of amino acid hydrazides and the desired substituted compounds.

Coupling of the mono-substituted hydrazine with the $N$-Boc amino acids was achieved, utilising standard peptide coupling reagents, in high yields to provide sufficient material to then allow coupling with the corresponding aryl carboxylic acids via the same procedure.

\subsection{Structure-Activity Relationship of Therapeutic Activity against Mtb}

Following the synthetic approach, the substituted amino acid hydrazides 6-37 were screened against drug-susceptible, INH or RIF mono-resistant strains of $M t b$ in a standard resazurin microtitre assay (REMA) [16,21]. Considering the entire results, all of the compounds broadly inhibited the growth of drug-susceptible and the mono-drug-resistant $M t b$ strains. In comparison with our previous 
studies using benzoxa-[2,1,3]-diazoles, we observed a level of antibacterial activity in the wild-type strain similar to or better than our lead compounds (Figure S1, Supporting Information). Notably, there was a greater antibacterial activity observed within the INH mono-resistant strain, implicating scope with this approach for the development of drugs with activity against drug-resistant $M t b$.

No discernible difference in antibacterial activity with Boc-protected compounds was observed between the 4-trifluoromethylphenyl alanine (Ala) hydrazide 6 or 4-trifluoromethylphenyl methionine (Met) hydrazide 7. However, encouragingly, the Boc-protected 3-chlorophenyl Ala and 3-chlorophenyl Met hydrazide $\mathbf{8}$ and $\mathbf{9}$ demonstrated an observable increase in activity relative to the 4-trifluorophenyl amino acid hydrazides 6-7 (Table 1).

Substitution of the Boc with aryl heterocycles that mimic INH and pyrazinamide, 10-17, in most cases, did not provide enhanced antibacterial activity (Table 1). Albeit this substitution in one case actually afforded no therapeutic activity $\mathbf{1 4}$. However, differential selectivity was noted, with excellent activity against INH-resistant $M t b$ exhibited with the 3-chlorophenyl Ala and Met hydrazide of pyridine 12-13 and pyrazine 16-17 (Table 1). This notable observation is promising towards the development of therapies for drug-resistant $M t b$, with significant potential that these compounds may employ a novel mechanism of action involving the circumventing of INH-activating enzyme, KatG, as the INH-resistant $M t b$ strain used in this study harboured an inactivating (Ser315 $\rightarrow$ Asp) in this protein, which was generated and identified in this study, analogous to previously published laboratory-generated and clinical strains [22].

An important criterion for any putative anti-TB drug is a demonstration of a clear therapeutic index between the concentration required to induce antibacterial activity and that which induces toxicity against the host system (i.e., macrophages and human tissueS). In this context, we set an arbitrary index of at least a 10-fold differential between the two outcomes. In the context of wild-type $M t b$ and RIF-resistant $M t b$, none of the molecules demonstrated any discernible differential between antibacterial activity and mammalian cell cytotoxicity. Interestingly and in contrast, several molecules demonstrated a $>10$-fold therapeutic index when evaluated against INH-resistant $M t b$. The Boc-protected 3-chlorophenyl Ala and Met hydrazides $\mathbf{8}$ and $\mathbf{9}$ gave excellent profiles, with a minimum inhibitory concentration (MIC) against INH-resistant $M t b$ of approximately $3 \mu \mathrm{M}$ and less than $10 \%$ toxicity against macrophages at $100 \mu \mathrm{M}$, indicating a 30-40-fold therapeutic index. Although the pyrazine 3-chlorophenyl Ala and Met hydrazides $\mathbf{1 6}$ and $\mathbf{1 7}$ also exhibited selectivity toward INH-resistant $M t b$, they demonstrated significant cytotoxicity against macrophages, implicating detrimental effects of the pyrimidine scaffold upon mammalian cells. The reasons for this are currently unclear.

The exploitation of the bedaquiline scaffolds, utilising the naphthalene and quinoline moieties (substituted at the 1-, 2- or 3- position with a carboxy group) 18-29, was evaluated as a strategy. Relative to the mono-substituted aryl heterocycles, this scaffold did not produce improved activity suggestive of further evaluation. Incorporation of the 2-substituted naphthalene moiety 18-21 produced a somewhat moderate, yet still disappointing, enhancement in anti-TB activity over 1- substituted naphthalene 22-25. Correspondingly, 3-substituted quinoline derivatives 26-29 displayed similar activity to the 2-naphthalene derivatives 18-21 with only the 4-trifluoromethylphenyl Met hydrazide 19 and 3-chlorophenyl Ala hydrazide 29 exhibiting anti-TB activity less than $50 \mu \mathrm{M}$. In support of these findings, a similar series of $\mathrm{N}$-(alkoxyphenyl)-2-hydroxynaphthalene-1-carboxamides and their positional isomers $\mathrm{N}$-(alkoxyphenyl)-1-hydroxynaphthalene-2-carboxamides have presented varied in vitro anti-TB activity (representative $12 \mu \mathrm{M}$ and $97 \mu \mathrm{M}$, respectively) [23]. Further to demonstrating the poor antibacterial activity, the inclusion of the bedaquiline scaffold also proved to be detrimental to mammalian cells, supporting the cessation of this scaffold as an approach. The only notable outcome from this series was the putative selectivity of 3-chlorophenyl Ala hydrazide $\mathbf{2 9}$ against INH-resistant $M t b$, with a MIC of $9 \mu \mathrm{M}$ versus $37 \mu \mathrm{M}$ against wild-type and RIF-resistant $M t b$ strains, albeit toxic against mammalian cells. This putative selectivity might be a consequence of bedaquiline inhibiting mycobacterial ATP synthase and counteracting the perturbation of the mycobacterial electron transport 
chain afforded by resistance to INH, as implicated in a recent study [24]. Further evaluation of this concept is, therefore, warranted.

Table 1. Results of synthesis of substituted amino acid hydrazides and the antibacterial activity and cytotoxicity.

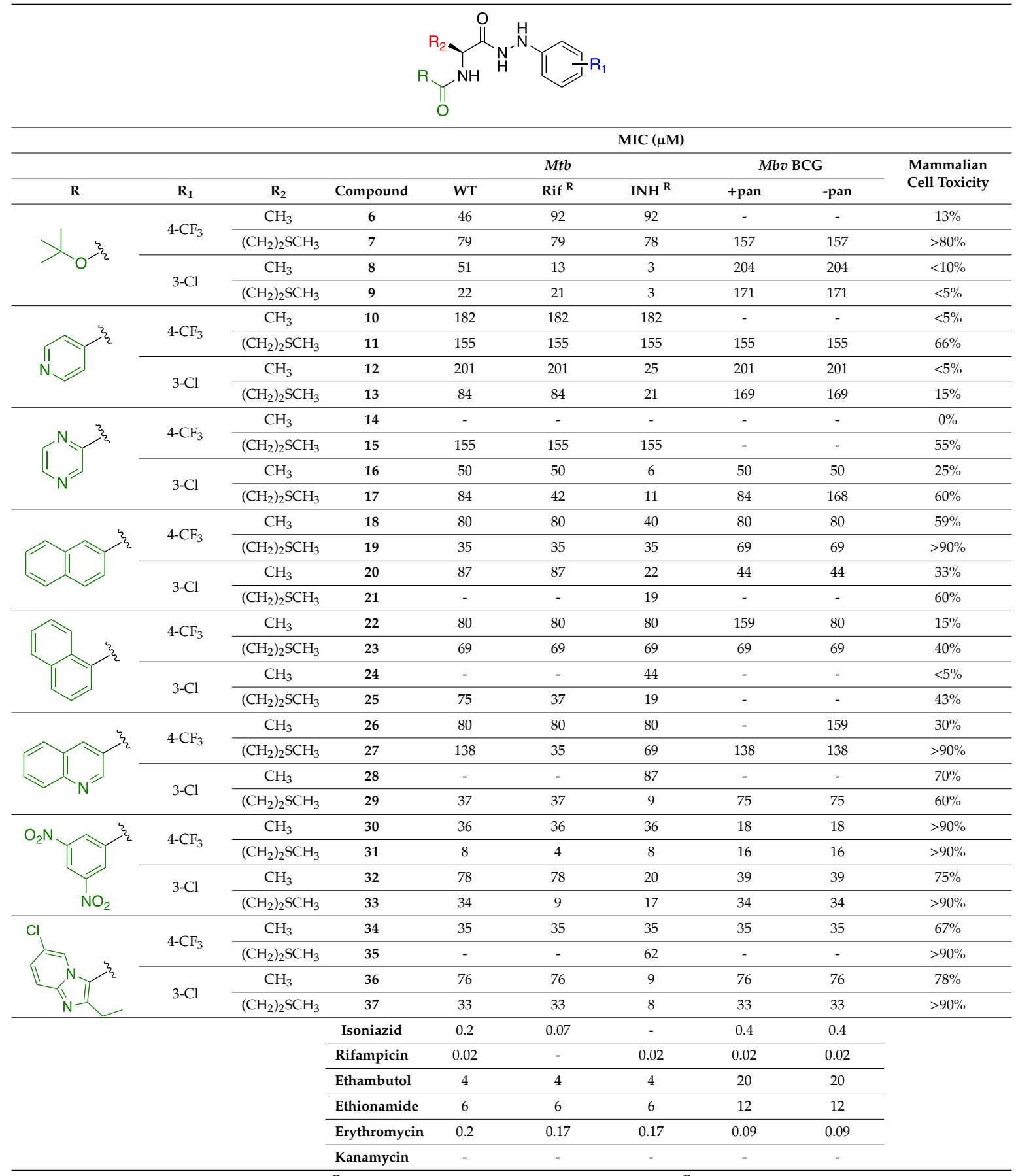

$\mathrm{WT}=$ Wild type $M t b$ strain; Rif ${ }^{\mathrm{R}}=$ Rifampicin resistant $M t b$ strain; $\mathrm{INH}^{\mathrm{R}}=$ Isoniazid resistant $M t b$ strain; + pan = Pantothenate present in growth media; - pan = Pantothenate not present in growth media; - = Not active at the maximum assay concentration $(64 \mu \mathrm{g} / \mathrm{mL})$.

Incorporation of 3,5-dinitrophenyl 30-33 resulted in viable antibacterial activity, with MIC values often $<50 \mu \mathrm{M}$ across all strains of $\mathrm{Mtb}$. Interestingly, differential selectivity towards drug-resistant strains was observed within this series. The 3-chlorophenyl Ala hydrazide of 3,5-dinitrophenyl 32 showed similar activity against wild-type and RIF-resistant $M t b$, with a 4 -fold greater activity against 
INH-resistant $M t b$. However, the 3-chlorophenyl Met hydrazide of 3,5-dinitrophenyl 33 showed increased activity relative to the Ala hydrazide against wild-type $M t b$ and a two-fold selectivity toward INH-resistant $M t b$ but surprisingly showed much greater activity against RIF-resistant $M t b$ with 4 -fold selectivity over that of wild-type $M t b$. This was the only compound in this series, demonstrating differential activity against RIF-resistant compared to wild-type $M t b$, the mechanism of which is as yet unexplored but worthy of further investigations. Mechanistically, it is highly likely that these compounds 30-33 induce a general nitric oxide stress response similar to that observed with the known anti-TB compounds-metronidazole, delamanid and pretomanid-involving metabolic activation by enzymes, such as deazaflavin-dependent nitroreductase or the pyruvate-ferredoxin oxidereductase system, based on the fact they include nitro containing groups and functionality [25,26]. Such a mode of action involving oxidative stress would explain the fact that this series was highly toxic against mammalian cells. However, unlike metronidazole, delamanid and pretomanid, the activation mechanism must involve an enzymatic pathway common to both bacteria and mammalian cells.

The Q203 analogues 34-37 were of particular interest with respect to the development of molecules, exploiting new therapeutic pathways and bypassing conventional drug resistance mechanisms, for the treatment of drug-resistant $M t b$. The imidazo[1,2-a]pyridine-3-carboxy moiety has been identified as the active component of several novel anti-tubercular drugs, including the pantothenate synthetase inhibitor GSK358607A and Q203 1, which targets the cytochrome bcc-aa3 supercomplex [27-29]. Although the 4-trifluoromethylphenyl imidazo[1,2-a]pyridine-3-carboxy-substituted hydrazides 34-35 demonstrated marginally better activity than their 3-chlorophenyl counterparts 36-37, no selectivity against drug-resistant strains of $M t b$ was observed with these compounds. With the 3-chlorophenyl imidazo[1,2-a]pyridine-3-carboxy-substituted hydrazides 36-37, the inclusion of Met over Ala resulted in a two-fold increase in antibacterial activity. In contrast to the 4-trifluoromethylphenyl-substituted hydrazides, the 3-chlorophenyl-substituted hydrazides showed exquisite selectivity toward INH-resistant $M t b$ with MIC values $<10 \mu \mathrm{M}$ for both the Ala 36 and Met 37 containing compounds. However, although showing potent antibacterial activity, all of the imidazo[1,2-a]pyridine-3-carboxy-substituted hydrazides exhibited significant toxicity against macrophages, implying their limited utility as viable drug molecules. Despite this, a greater understanding of the structure-activity relationship (SAR) and identification of the targets of these compounds is an important concept in improving our understanding of principles associated with their selectivity towards drug-resistant $M t b$.

With respect to the imidazo[1,2-a]pyridine-3-carboxy-derived hydrazides 34-37, it was conceivable that the target of these compounds could include either the cytochrome supercomplex of proteins, as is the case with Q203 1, or pantothenate synthetase, the reported target of GSK358607A. Nevertheless, our results implied that the target was unlikely to be pantothenate synthetase since the $M t b \mathrm{mc}^{2} 7000$ strains used in this study possessed $\triangle$ panCD gene deletions and relied on media supplementation with pantothenate for survival and growth, thereby abrogating dependency for growth upon this enzyme. Inclusion of the imidazo[1,2-a]pyridine-3-carboxy moiety resulted in equivalent or greater activity than the initial Boc-protected compounds 6-9. To further confirm this hypothesis, the activity of compounds 34-37 was evaluated against Mycobacterium bovis BCG as this constitutively expressed the pantothenate pathway. No difference in activity was detected with these compounds when in the presence or absence of pantothenate in the growth media with this mycobacterium, reinforcing pantothenate synthetase as unlikely to be the therapeutic target for these compounds. Furthermore, this target would also not explain the selectivity of these compounds for INH-resistant $M t b$.

A more likely mechanism and one which provides a rationale for the increased selectivity of the 3-chlorophenyl imidazo[1,2-a]pyridine-3-carboxy-substituted hydrazides 36-37 towards INH-resistant $M t b$ is one analogous to that of Q203. This drug targets the cytochrome bcc-aa3 supercomplex and disrupts the ETC in mycobacteria [30]. Recently, in addition to inhibiting mycolic acid synthesis, the bactericidal activity of INH has also been linked to ATP production, increased oxygen consumption and subsequently perturbation of the mycobacterial ETC [24]. In this 
context, defects in NADH dehydrogenase and concomitantly ETC have been shown to confer resistance to INH in mycobacteria [31]. Further studies are thereby warranted to evaluate the involvement of this pathway in terms of conferring therapeutic selectivity of 3-chlorophenyl imidazo[1,2-a]pyridine-3-carboxy-substituted hydrazides towards INH-resistant $M t b$. In addition, SAR studies to address the selectivity of the 3-chlorophenyl-but not the 4-trifluoromethylphenyl-substituted hydrazides are also important.

\section{Materials and Methods}

All reactions were carried out under a nitrogen atmosphere in glassware dried in the oven overnight $\left(>75^{\circ} \mathrm{C}\right)$ or under high vacuum by a heat-gun unless otherwise stated. Ether refers to diethyl ether. Solvents were obtained dry from Sigma Aldrich, Gillingham, UK or another appropriate chemical supplier and used as supplied. In cases where mixtures of solvents were utilised, the ratios refer to the volumes used. Reagents were used as supplied unless otherwise stated. Flash chromatography was carried out using silica gel 40-63 $\mu$ 60A. Analytical thin layer chromatography (TLC) was performed using precoated aluminium or glass-backed plates (silica gel $60 \AA \mathrm{F}_{254}$ ) and visualised by UV radiation at $254 \mathrm{~nm}$, or by staining with phosphomolybdic acid in ethanol or potassium permanganate in water. All melting points were determined using a Gallenkamp melting point apparatus and are uncorrected. Infrared spectra were recorded using a Diamond ATR (attenuated total reflection) accessory (Golden Gate) or as a solution in chloroform via transmission IR cells on a Bruker Tension 5 spectrometer. ${ }^{1} \mathrm{H}$ NMR spectra were recorded in deutero solvent on Bruker Avance III 300, Bruker Avance II 400, Bruker Avance III HD 500 or Bruker Avance III HD 700 instruments and are reported as follows; chemical shift $\delta(\mathrm{ppm})$ (number of protons, multiplicity, coupling constant $J(\mathrm{~Hz})$, assignment). Residual protic solvent $\mathrm{CHCl}_{3}\left(\delta_{\mathrm{H}}=7.26\right)$ or DMSO $\left(\delta_{\mathrm{H}}=2.50\right)$ were used as the internal reference. ${ }^{13} \mathrm{C}$ NMR spectra were recorded using the central resonance of $\mathrm{CDCl}_{3}\left(\delta_{\mathrm{C}}=77.0 \mathrm{ppm}\right)$ or DMSO $\left(\delta_{\mathrm{C}}=39.5 \mathrm{ppm}\right)$ as the internal reference. All chemical shifts are quoted in parts per million relative to tetramethylsilane $\left(\delta_{\mathrm{H}}=0.00 \mathrm{ppm}\right)$ and coupling constants are given in Hertz to the nearest $1 \mathrm{~Hz}$. Assignment of spectra were carried out using COSY, HSQC, HMBC and NOESY experiments and all ${ }^{1} \mathrm{H}$ and ${ }^{13} \mathrm{C}$ NMR for new compounds are provided for reference (Figure S2, Supporting Information). Electrospray mass spectra (ES) were obtained on a Micromass LCT mass spectrometer. High resolution mass spectra (HRMS) were obtained using a Thermo LTQ mass spectrometer (ES).

\subsection{Chemistry}

\subsubsection{Standard Procedure A: Synthesis of Boc Amino Acid Hydrazides*}

A solution of $\mathrm{N}$-Boc amino acid $(1.0 \mathrm{~g}, 5.5 \mathrm{mmol})$ in dichloromethane (DCM) $(25 \mathrm{~mL})$ was treated with $N, N$-Diisopropylethylamine (DIPEA) (1.2 equiv.) and $N$-aryl hydrazine (1.2 equiv.), followed by 4-(4,6-dimethoxy-1,3,5-triazin-2-yl)-4-methyl-morpholinium chloride (DMTMM) (1.2 equiv.), and the solution was stirred at room temperature for $3 \mathrm{~h}$. The reaction was mixed with DCM $(20 \mathrm{~mL})$, and the organic layer was washed with sat. aq. $\mathrm{NH}_{4} \mathrm{Cl}(20 \mathrm{~mL})$, sat. aq. $\mathrm{NaHCO}_{3}(20 \mathrm{~mL})$ and brine $(20 \mathrm{~mL})$. The organic layer was dried over $\mathrm{MgSO}_{4}$, filtered, evaporated and dried in vacuo to afford the desired $N$-Boc amino acid hydrazides.

*Compound 6 synthesis is previously reported [16].

\subsubsection{Standard Procedure B: Creation of New Amino Acid Hydrazides}

\section{Stage 1}

The corresponding $N$-Boc amino acid hydrazides $(0.10 \mathrm{~g}, 0.29 \mathrm{mmol})$ were mixed with $4 \mathrm{M} \mathrm{HCl}$ in dioxane $(3 \mathrm{~mL})$ and stirred at room temperature for $1 \mathrm{~h}$ under nitrogen. The solvent was then removed in vacuo, and the resultant oil was dissolved in DCM $(5 \mathrm{~mL})$ and evaporated three times 
to afford the desired deprotected amino acid hydrazide, which was used directly in stage 2 without further purification.

Stage 2

Crude deprotected amino acid hydrazide $(0.29 \mathrm{mmol})$ was dissolved in DCM $(5 \mathrm{~mL})$ and treated successively with the aryl carboxylic acid (1.3 equiv.) and DIPEA (2.0 equiv.), followed by DMTMM (1.2 equiv.) at room temperature under nitrogen. The reaction was stirred for $3 \mathrm{~h}$, then diluted with DCM $(15 \mathrm{~mL})$. The organic layer was washed with sat. aq. $\mathrm{NH}_{4} \mathrm{Cl}(20 \mathrm{~mL})$, sat. aq. $\mathrm{NaHCO}_{3}(20$ $\mathrm{mL}$ ) and brine $(20 \mathrm{~mL})$. The organic layer was dried over $\mathrm{MgSO}_{4}$, filtered, evaporated and dried in vacuo. The resultant solid was triturated with $\mathrm{CHCl}_{3}$ to afford the desired $\mathrm{N}$-substituted amino acid hydrazide.

\subsubsection{Boc-Protected Compounds}

tert-butyl $N-\left[(1 S)-3-(m e t h y l s u l f a n y l)-1-\left\{N^{\prime}\right.\right.$-[4-(trifluoromethyl)phenyl]hydrazine carbonyl\}propyl]carbamate, 7, following the standard procedure A, Boc-Met-OH $(0.2 \mathrm{~g}, 0.8 \mathrm{mmol})$ and 4-trifluoromethylphenylhydrazine were transformed following flash chromatography (DCM/EtOH/ sat. aq. $\mathrm{NH}_{3}$ [400:8:1], [200:8:1] and [100:8:1]) to afford the title compound as a pale yellow solid $(0.14 \mathrm{~g}, 44 \%) ; \mathrm{R}_{\mathrm{f}} 0.32(\mathrm{DCM} / \mathrm{EtOH} / \mathrm{sat}$. aq. $\mathrm{NH}_{3}$ [200:8:1]); m.p. $102-108{ }^{\circ} \mathrm{C}$; $v_{\max }$ (ATR) 3321, 2984, 1680, 1662, 1515, 1327, 1306, 1156, 1102, 830, $589 \mathrm{~cm}^{-1} ; \delta_{\mathrm{H}}\left(700 \mathrm{MHz}, \mathrm{CDCl}_{3}\right) 8.82(1 \mathrm{H}, \mathrm{s}, \mathrm{CONHNH}), 7.41(2 \mathrm{H}, \mathrm{d}, J$ 8, $\mathrm{Ar}-\mathrm{H}), 6.81(2 \mathrm{H}, \mathrm{d}, J$ 8, Ar-H), $6.53(1 \mathrm{H}, \mathrm{s}, \mathrm{CONHNH}), 5.43(1 \mathrm{H}, \mathrm{d}, J$ 8, NHCHCO), $4.44(1 \mathrm{H}, \mathrm{q}, J$ 7, NHCHCO), $2.56(2 \mathrm{H}$, $\mathrm{t}, J$ 7, $\left.\mathrm{NHCH}\left(\mathrm{CH}_{2} \mathrm{CH}_{2} \mathrm{SCH}_{3}\right)\right), 2.09-2.14\left(5 \mathrm{H}, \mathrm{m}, \mathrm{NHCH}\left(\mathrm{CH}_{2} \mathrm{CH}_{2} \mathrm{SCH}_{3}\right), \mathrm{NHCH}\left(\mathrm{CH}_{2} \mathrm{CH}_{2} \mathrm{SCH}_{3}\right)\right), 1.49$ $\left(9 \mathrm{H}, \mathrm{s},\left(\mathrm{C}\left(\mathrm{CH}_{3}\right)_{3}\right) ; \delta_{\mathrm{C}}\left(700 \mathrm{MHz}, \mathrm{CDCl}_{3}\right) 172.4(\mathrm{CHCONH}), 156.1\left(\mathrm{NHCOOC}\left(\mathrm{CH}_{3}\right)_{3}\right), 150.5(\mathrm{Ar}-\mathrm{C}), 126.3\right.$ $(\mathrm{Ar}-\mathrm{C}), 124.2\left(1 \mathrm{C}, \mathrm{q},{ }^{1} J_{\mathrm{C}-\mathrm{F}} 270, \mathrm{CF}_{3}\right), 122.7\left(1 \mathrm{C}, \mathrm{q},{ }^{2} \mathrm{~J}_{\mathrm{C}-\mathrm{F}} 32, i p s o-\mathrm{Ar}^{-\mathrm{CCF}_{3}}\right), 112.7$ (Ar-C), 80.9 (NHCOO-C), $51.9(\mathrm{NHCHCO}), 30.9\left(\mathrm{NHCH}\left(\mathrm{CH}_{2} \mathrm{CH}_{2} \mathrm{SCH}_{3}\right)\right), 30.24\left(\mathrm{NHCH}\left(\mathrm{CH}_{2} \mathrm{CH}_{2} \mathrm{SCH}_{3}\right)\right), 28.3\left(\mathrm{NHCOOC}\left(\mathrm{CH}_{3}\right)_{3}\right)$, $15.4\left(\mathrm{NHCH}\left(\mathrm{CH}_{2} \mathrm{CH}_{2} \mathrm{SCH}_{3}\right)\right) ; \mathrm{m} / z\left(\mathrm{ES}^{+}\right) 408\left(\mathrm{MH}^{+}\right) ; \mathrm{HRMS}\left(\mathrm{ES}^{+}\right)$found $\mathrm{MH}^{+}, 408.1573\left(\mathrm{C}_{17} \mathrm{H}_{25} \mathrm{~F}_{3} \mathrm{~N}_{3} \mathrm{O}_{3} \mathrm{~S}\right.$ requires 408.1569$)$.

tert-butyl (S)-(1-(2-(3-chlorophenyl)hydrazineyl)-1-oxopropan-2-yl)carbamate, 8, following the standard procedure A, Boc-Ala-OH $(1.0 \mathrm{~g}, 5.3 \mathrm{mmol})$ and 3-chlorophenylhydrazine were transformed to afford the title compound as a deep-yellow solid (1.6 g, 97\%); $\mathrm{R}_{\mathrm{f}} 0.46\left(\mathrm{DCM} / \mathrm{EtOH} / \mathrm{sat}\right.$. aq. $\mathrm{NH}_{3}$ [200:8:1]); m.p. $110-114{ }^{\circ} \mathrm{C} ; v_{\max }$ (ATR) $3428,3278,3078,2979,2933,1678,1504,1367,1158 \mathrm{~cm}^{-1} ; \delta_{\mathrm{H}}$ $\left(400 \mathrm{MHz}, \mathrm{CDCl}_{3}\right) 8.44(1 \mathrm{H}, \mathrm{bs}, \mathrm{CONHNH}), 7.03(1 \mathrm{H}, \mathrm{t}, J$ 8, $\mathrm{Ar}-\mathrm{H}), 6.76(1 \mathrm{H}, \mathrm{d}, J$ J, $\mathrm{Ar}-\mathrm{CH}), 6.71(1 \mathrm{H}, \mathrm{t}, J 2$, $\left.\mathrm{Ar}-\mathrm{CH}), 6.60(1 \mathrm{H}, \mathrm{d}, \mathrm{J} 8, \mathrm{Ar}-\mathrm{CH}), 5.07(1 \mathrm{H}, \mathrm{bs}, \mathrm{CONHCH}), 4.22-4.19(1 \mathrm{H}, \mathrm{m}, \mathrm{CH}), 1.39\left(9 \mathrm{H}, \mathrm{s},\left(\mathrm{C}_{\left(\mathrm{CH}_{3}\right)}\right)_{3}\right)\right)$, $1.31\left(3 \mathrm{H}, \mathrm{d}, \mathrm{J}\right.$ 7, $\left.\mathrm{NHCHCH}_{3} \mathrm{C}=\mathrm{O}\right) ; \delta_{\mathrm{C}}\left(400 \mathrm{MHz}, \mathrm{CDCl}_{3}\right) 192.7(\mathrm{OC}=\mathrm{ONH}), 173.0(\mathrm{CHC}=\mathrm{ONHNH})$, $155.9\left(\left(\mathrm{CH}_{3}\right)_{3} \mathrm{COC}=\mathrm{O}\right), 149.1(\mathrm{Ar}-\mathrm{C}), 135.0(\mathrm{Ar}-\mathrm{C}), 130.2(\mathrm{Ar}-\mathrm{CH}), 121.0(\mathrm{Ar}-\mathrm{C}), 113.4(\mathrm{Ar}-\mathrm{CH}), 111.69$ $(\mathrm{Ar}-\mathrm{CH}), 48.7(\mathrm{NHCHC}=\mathrm{O}), 28.3\left(\mathrm{C}\left(\mathrm{CH}_{3}\right)_{3}\right), 17.5\left(\mathrm{NHCH}\left(\mathrm{CH}_{3}\right) \mathrm{CO}\right) ; \mathrm{m} / z\left(\mathrm{ES}^{+}\right) 314\left(\left[{ }^{35} \mathrm{Cl} \mathrm{MH}^{+}\right), 316\right.$ $\left(\left[{ }^{37} \mathrm{Cl}\right] \mathrm{MH}^{+}\right)$; HRMS $\left(\mathrm{ES}^{+}\right)$found $\left(\left[{ }^{35} \mathrm{Cl}\right] \mathrm{MH}^{+}\right), 314.1271\left(\mathrm{C}_{14} \mathrm{H}_{21} \mathrm{~N}_{3} \mathrm{O}_{3}{ }^{35} \mathrm{Cl}\right.$ requires 314.1278$)$.

tert-Butyl $N$-[(1S)-1-[N'-(3-chlorophenyl)hydrazinecarbonyl]-3-(methylsulfanyl)propyl] carbamate, 9, following the standard procedure A, Boc-Met-OH $(0.2 \mathrm{~g}, 0.802 \mathrm{mmol})$ and 3-chlorophenylhydrazine were transformed following flash chromatography $\left(\mathrm{DCM} / \mathrm{EtOH} /\right.$ sat. aq. $\mathrm{NH}_{3}$ [400:8:1] and [200:8:1]) to afford the title compound as a pale yellow solid $(0.16 \mathrm{~g}, 53 \%) ; \mathrm{R}_{\mathrm{f}} 0.30\left(\mathrm{DCM} / \mathrm{EtOH} /\right.$ sat. aq. $\mathrm{NH}_{3}$ [200:8:1]); m.p. $94-97^{\circ} \mathrm{C} ; v_{\max }$ (ATR) 3331, 3272, 2985, 1683, 1658, 1521, 1163, $768 \mathrm{~cm}^{-1} ; \delta_{\mathrm{H}}(700 \mathrm{MHz}$, $\left.\mathrm{CDCl}_{3}\right) 8.73(1 \mathrm{H}, \mathrm{s}, \mathrm{CONHNH}), 7.10(1 \mathrm{H}, \mathrm{t}, J$ 8, Ar-H), $6.85(1 \mathrm{H}, \mathrm{d}, J$ 8, Ar-H), $6.80(1 \mathrm{H}, \mathrm{s}, \mathrm{Ar}-\mathrm{H}), 6.67$ $(1 \mathrm{H}, \mathrm{d}, J$ 8, $\mathrm{Ar}-\mathrm{H}), 6.34(1 \mathrm{H}, \mathrm{bs}, \mathrm{CONHNH}), 5.42(1 \mathrm{H}, \mathrm{d}, J$ 8, NHCHCO $), 4.43(1 \mathrm{H}, \mathrm{q}, J$ 7, NHCHCO), $2.57\left(2 \mathrm{H}, \mathrm{t}, J\right.$ 7, $\left.\mathrm{NHCHCH}_{2} \mathrm{CH}_{2} \mathrm{SCH}_{3}\right), 2.09-2.13\left(5 \mathrm{H}, \mathrm{m}, \mathrm{NHCHCH}_{2} \mathrm{CH}_{2} \mathrm{SCH}_{3}, \mathrm{NHCHCH}_{2} \mathrm{CH}_{2} \mathrm{SCH}_{3}\right)$, $1.47\left(9 \mathrm{H}, \mathrm{s}, \mathrm{C}\left(\mathrm{CH}_{3}\right)_{3}\right) ; \delta_{\mathrm{C}}\left(700 \mathrm{MHz}, \mathrm{CDCl}_{3}\right) 172.2(\mathrm{CHCONH}), 156.9\left(\mathrm{NHCOO}\left(\mathrm{CH}_{3}\right)_{3}\right), 149.0(\mathrm{Ar}-\mathrm{C})$, 134.9 (Ar-C), $130.2(\mathrm{Ar}-\mathrm{C}), 121.0(\mathrm{Ar}-\mathrm{C}), 113.4(\mathrm{Ar}-\mathrm{C}), 111.6(\mathrm{Ar}-\mathrm{C}), 80.8\left(\mathrm{NHCOOC}\left(\mathrm{CH}_{3}\right)_{3}\right), 51.9$

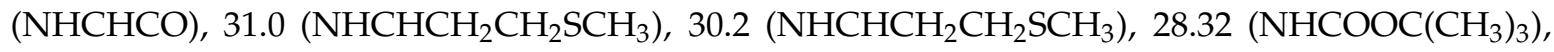


$15.5\left(\mathrm{NHCHCH}_{2} \mathrm{CH}_{2} \mathrm{SCH}_{3}\right) ; \mathrm{m} / z\left(\mathrm{ES}^{+}\right) 374\left(\left[{ }^{35} \mathrm{Cl}\right] \mathrm{MH}^{+}\right), 376\left(\left[{ }^{37} \mathrm{Cl}\right] \mathrm{MH}^{+}\right), 396\left(\left[{ }^{35} \mathrm{Cl}\right) \mathrm{MNa}^{+}\right), 398$ $\left(\left[{ }^{37} \mathrm{Cl}\right] \mathrm{MNa}^{+}\right)$; HRMS $\left(\mathrm{ES}^{+}\right)$found $\left(\left[{ }^{35} \mathrm{Cl}\right] \mathrm{MNa}^{+}\right), 396.1141\left(\mathrm{C}_{16} \mathrm{H}_{24} \mathrm{~N}_{3} \mathrm{O}_{3} \mathrm{~S}^{35} \mathrm{ClNa}\right.$ requires 396.1125$)$.

\subsubsection{Final Compounds}

(S)-N-(1-Oxo-1-(2-(4-(trifluoromethyl)phenyl)hydrazineyl)propan-2-yl)isonicotinamide, 10, following the standard procedure B, tert-butyl (S)-(1-oxo-1-(2-(4-(trifluoromethyl) phenyl)hydrazineyl)propan-2-yl) carbamate $(0.10 \mathrm{~g}, 0.30 \mathrm{mmol})$ and isonicotinic acid were transformed to afford the title compound as a pale-yellow solid (76 mg, 72\%); $\mathrm{R}_{\mathrm{f}} 0.15\left(\mathrm{DCM} / \mathrm{EtOH} /\right.$ sat. aq. $\mathrm{NH}_{3}$ [200:8:1]); $v_{\max }$ (ATR) 3261 (N-H), 3095, $2973(\mathrm{C}-\mathrm{H}), 1638(\mathrm{C}=\mathrm{O})$, 1617, 1536, $1327(\mathrm{C}-\mathrm{F}), 1158(\mathrm{C}-\mathrm{N}), 1102,1065(\mathrm{C}-\mathrm{F}) \mathrm{cm}^{-1} ; \delta_{\mathrm{H}}$ (400 MHz, DMSO-d 6 ) 10.05 (1H, s, CONHNH), 8.98 (1H, d, J 7, PyCONH), 8.73 (2H, d, J 6, Py-H), 8.39 $(1 \mathrm{H}, \mathrm{bs}, \mathrm{CONHNH}), 7.82(2 \mathrm{H}, \mathrm{d}, J$ 6 , Py-H), $7.44(2 \mathrm{H}, \mathrm{d}, J$ 8, Ar-H), $6.83(2 \mathrm{H}, \mathrm{d}, J$ 8, Ar-H), $4.50(1 \mathrm{H}$, $\left.\mathrm{m}, \mathrm{NHCH}\left(\mathrm{CH}_{3}\right) \mathrm{CO}\right), 1.44\left(3 \mathrm{H}, \mathrm{d}, J\right.$ 7, $\left.\mathrm{NHCH}\left(\mathrm{CH}_{3}\right) \mathrm{CO}\right) ; \delta_{\mathrm{C}}\left(100 \mathrm{MHz}, \mathrm{DMSO}-\mathrm{d}_{6}\right) 172.6(\mathrm{CONHNH})$, 165.4 (PyCONH), 152.9 (ipso-Ar-C), 150.6 (Py-C), 141.4 (ipso-Py-C), 126.6 (Ar-C), 124.4 (1C, q, ${ }^{1} J_{\mathrm{C}-\mathrm{F}}$ 270, $\left.\mathrm{CF}_{3}\right), 122.0$ (Py-C), $118.5\left(1 \mathrm{C}, \mathrm{q},{ }^{2} J_{\mathrm{C}-\mathrm{F}} 31\right.$, ipso-Ar- $\left.\mathrm{CCF}_{3}\right), 111.9(\mathrm{Ar}-\mathrm{C}), 48.7\left(\mathrm{NHCH}\left(\mathrm{CH}_{3}\right) \mathrm{CO}\right), 18.0$ $\left(\mathrm{NHCH}\left(\mathrm{CH}_{3}\right) \mathrm{CO}\right) ; \delta_{\mathrm{F}}\left(282 \mathrm{MHz}, \mathrm{DMSO}-\mathrm{d}_{6}\right)-59.28\left(3 \mathrm{~F}, \mathrm{~s}, \mathrm{CF}_{3}\right) ; \mathrm{m} / z\left(\mathrm{ES}^{+}\right) 353\left(\mathrm{MH}^{+}\right) ; \mathrm{HRMS}\left(\mathrm{ES}^{+}\right)$ found $\mathrm{MH}^{+}, 353.1237\left(\mathrm{C}_{16} \mathrm{H}_{16} \mathrm{~F}_{3} \mathrm{~N}_{4} \mathrm{O}_{2}\right.$ requires 353.1225).

(S)-N-(4-(methylthio)-1-oxo-1-(2-(4-(trifluoromethyl)phenyl)hydrazineyl)butan-2-yl)isonicotinamide, 11, following the standard procedure B, tert-Butyl $N-\left[(1 S)-3\right.$-(methylsulfanyl)-1- $\left\{N^{\prime}\right.$-[4-(trifluoromethyl) phenyl]hydrazine carbonyl propyl]carbamate $(0.14 \mathrm{~g}, 0.41 \mathrm{mmol})$ and isonicotinic acid were transformed to afford the title compound as a white solid (108 mg, 64\%); m.p. $179-181^{\circ} \mathrm{C} ; v_{\max }$ (ATR) $3264,1665,1639,1615,1531,1480,1410,1323,1236,1158,1105,1065,1010,878,836,757 \mathrm{~cm}^{-1} ; \delta_{\mathrm{H}}$ (300 MHz, DMSO-d 6$) 10.12(1 \mathrm{H}, \mathrm{s}, \mathrm{CONHNH}), 8.98(1 \mathrm{H}, \mathrm{d}, J$ 7, PyCONH), $8.74(2 \mathrm{H}, \mathrm{d}, J 6$ 6, Py-H), $8.43(1 \mathrm{H}, \mathrm{bs}, \mathrm{CONHNH}), 7.84(2 \mathrm{H}, \mathrm{d}, J$ 6, Py-H), $7.45(2 \mathrm{H}, \mathrm{d}, J$ 8, Ar-H), $6.82(2 \mathrm{H}, \mathrm{d}, J$ 8, Ar-H), $4.61\left(1 \mathrm{H}, \mathrm{m}, \mathrm{NHCH}\left(\mathrm{CH}_{2} \mathrm{CH}_{2} \mathrm{SCH}_{3}\right)\right), 2.67-2.53\left(2 \mathrm{H}, \mathrm{m}, \mathrm{NHCH}\left(\mathrm{CH}_{2} \mathrm{CH}_{2} \mathrm{SCH}_{3}\right)\right), 2.15-2.09(5 \mathrm{H}, \mathrm{m}$, $\mathrm{NHCH}\left(\mathrm{CH}_{2} \mathrm{CH}_{2} \mathrm{SCH}_{3}\right) ; \delta_{\mathrm{C}}\left(75 \mathrm{MHz}, \mathrm{DMSO}-\mathrm{d}_{6}\right) 171.7(\mathrm{CONHNH}), 165.3(\mathrm{PyCONH}), 150.6$ (Py-C), 126.6 (Ar-C), $122.1(\mathrm{Py}-\mathrm{C}), 111.9(\mathrm{Ar}-\mathrm{C}), 52.3\left(\mathrm{NHCH}\left(\mathrm{CH}_{2} \mathrm{CH}_{2} \mathrm{SCH}_{3}\right)\right), 31.3\left(\mathrm{NHCH}\left(\mathrm{CH}_{2} \mathrm{CH}_{2} \mathrm{SCH}_{3}\right)\right)$, $30.4\left(\mathrm{NHCH}\left(\mathrm{CH}_{2} \mathrm{CH}_{2} \mathrm{SCH}_{3}\right)\right)$, $15.1\left(\mathrm{NHCH}\left(\mathrm{CH}_{2} \mathrm{CH}_{2} \mathrm{SCH}_{3}\right)\right)$; $\delta_{\mathrm{F}}\left(282 \mathrm{MHz}, \mathrm{DMSO}-\mathrm{d}_{6}\right)-59.28(3 \mathrm{~F}, \mathrm{~s}$, $\left.\mathrm{CF}_{3}\right) ; m / z\left(\mathrm{ES}^{+}\right) 413\left(\mathrm{MH}^{+}\right) ; \mathrm{HRMS}\left(\mathrm{ES}^{+}\right)$found $\mathrm{MH}^{+}, 413.1261\left(\mathrm{C}_{18} \mathrm{H}_{20} \mathrm{~N}_{4} \mathrm{O}_{2} \mathrm{~S}\right.$ requires 413.1259).

(S)-N-(1-(2-(3-chlorophenyl)hydrazineyl)-1-oxopropan-2-yl)isonicotinamide, 12, following the standard procedure B, tert-butyl (S)-(1-(2-(3-chlorophenyl)hydrazineyl)-1-oxopropan-2-yl)carbamate (0.10 g, $0.32 \mathrm{mmol})$ and isonicotinic acid were transformed to afford the title compound as a white solid $(60 \mathrm{mg}$, 59\%); $\mathrm{R}_{\mathrm{f}} 0.13$ (DCM/EtOH/ sat. aq. $\mathrm{NH}_{3}$ [200:8:1]); $v_{\max }$ (ATR) 3302, $3254(\mathrm{~N}-\mathrm{H}), 3035(\mathrm{Ar}-\mathrm{CH}), 2983$, $2930(\mathrm{C}-\mathrm{H}), 1668,1638(\mathrm{C}=\mathrm{O})$ 1599, 1537, $1511(\mathrm{C}=\mathrm{C}) 1106 \mathrm{~cm}^{-1}$; $\delta_{\mathrm{H}}\left(400 \mathrm{MHz}, \mathrm{DMSO}-\mathrm{d}_{6}\right) 9.97(1 \mathrm{H}, \mathrm{s}$, CONHNH), $8.99(1 \mathrm{H}, \mathrm{d}, J$ 7, Py-CONH), $8.75(2 \mathrm{H}, \mathrm{d}, J$ 6, Py-H), $8.08(1 \mathrm{H}, \mathrm{s}, \mathrm{CONHNH}), 7.84(2 \mathrm{H}, \mathrm{d}, J$, Py-H), $7.14\left(1 \mathrm{H}, \mathrm{t}, J\right.$ 8, Ar-H), 6.75-6.66 (3H, m, Ar-H), $4.53\left(1 \mathrm{H}, \mathrm{m}, \mathrm{NHCH}\left(\mathrm{CH}_{3}\right) \mathrm{CO}\right), 1.44(3 \mathrm{H}, \mathrm{d}, J$, $\left.\mathrm{NHCH}\left(\mathrm{CH}_{3}\right) \mathrm{CO}\right) ; \delta_{\mathrm{C}}\left(100 \mathrm{MHz}, \mathrm{DMSO}-\mathrm{d}_{6}\right)$ 172.6 (CONHNH), 165.4 (Py-CO), 151.3 (ipso-Ar-C), 150.6 (Py-C), 141.3 (ipso-Py-C), 133.9 (ipso-Ar-C), 130.5 (Ar-C), 122.0 (Py-C), 118.3 (Ar-C), 111.9 (Ar-C), 111.3 $(\mathrm{Ar}-\mathrm{C}), 48.7\left(\mathrm{NHCH}\left(\mathrm{CH}_{3}\right) \mathrm{CO}\right), 17.9\left(\mathrm{NHCH}\left(\mathrm{CH}_{3}\right) \mathrm{CO}\right) ; \mathrm{m} / z\left(\mathrm{ES}^{+}\right) 319\left(\left[{ }^{37} \mathrm{Cl}\right] \mathrm{MH}^{+}\right), 321\left(\left[{ }^{35} \mathrm{Cl}^{-} \mathrm{MH}^{+}\right)\right.$; HRMS $\left(\mathrm{ES}^{+}\right)$found $\left[{ }^{35} \mathrm{Cl}\right] \mathrm{MH}^{+}, 319.0962\left(\mathrm{C}_{15} \mathrm{H}_{16} \mathrm{~N}_{4} \mathrm{O}_{2}{ }^{35} \mathrm{Cl}\right.$ requires 319.0962).

(S)-N-(1-(2-(3-chlorophenyl)hydrazineyl)-4-(methylthio)-1-oxobutan-2-yl)isonicotinamide, 13, following the standard procedure B, tert-Butyl N-[(1S)-1-[N'-(3-chlorophenyl)hydrazinecarbonyl]-3-(methylsulfanyl) propyl] carbamate $(0.35 \mathrm{~g}, 1.13 \mathrm{mmol})$ and isonicotinic acid were transformed to afford the title compound as a brown solid (113 mg, 26\%); m.p. 78-81 ${ }^{\circ} \mathrm{C}$; $v_{\max }$ (ATR) 3259, 2919, 1659, 1622, 1597, 0521, 1475, 1407, 1385, 1310, 1229, 1151, 1096, 1065, 992, $834 \mathrm{~cm}^{-1} ; \delta_{\mathrm{H}}\left(300 \mathrm{MHz}, \mathrm{DMSO}-\mathrm{d}_{6}\right) 10.03(1 \mathrm{H}, \mathrm{s}$, CONHNH), 8.98 (1H, d, J 7, PyCONH), 8.75 (2H, d, J 6, Py-H), 8.09 (1H, bs, CONHNH), 7.84 (2H, d, J 6, Py-H), $7.15\left(1 \mathrm{H}, \mathrm{t}, J\right.$ 8, Ar-H), 6.78-6.62 (3H, m, Ar-H), $4.60\left(1 \mathrm{H}, \mathrm{m}, \mathrm{NHCH}\left(\mathrm{CH}_{2} \mathrm{CH}_{2} \mathrm{SCH}_{3}\right)\right), 2.67-2.53$ $\left(2 \mathrm{H}, \mathrm{m}, \mathrm{NHCH}\left(\mathrm{CH}_{2} \mathrm{CH}_{2} \mathrm{SCH}_{3}\right)\right), 2.09-1.98\left(5 \mathrm{H}, \mathrm{m}, \mathrm{NHCH}\left(\mathrm{CH}_{2} \mathrm{CH}_{2} \mathrm{SCH}_{3}\right) ; \delta_{\mathrm{C}}\left(75 \mathrm{MHz}, \mathrm{DMSO}-\mathrm{d}_{6}\right)\right.$ 
171.6 (CONHNH), 165.8 (PyCONH), 151.3 (ipso-Ar-C), 150.6 (Py-C), 141.4 (ipso-Ar-C), 134.0 (ipso-Ar-C), 131.3 (ipso-Ar-C), $130.8(\mathrm{Ar}-\mathrm{C}), 122.1(\mathrm{Py}-\mathrm{C}), 118.3(\mathrm{Ar}-\mathrm{C}), 111.9(\mathrm{Ar}-\mathrm{C}), 52.3\left(\mathrm{NHCH}\left(\mathrm{CH}_{2} \mathrm{CH}_{2} \mathrm{SCH}_{3}\right)\right.$ ), $31.2\left(\mathrm{NHCH}\left(\mathrm{CH}_{2} \mathrm{CH}_{2} \mathrm{SCH}_{3}\right)\right), 30.4\left(\mathrm{NHCH}\left(\mathrm{CH}_{2} \mathrm{CH}_{2} \mathrm{SCH}_{3}\right)\right), 15.1\left(\mathrm{NHCH}\left(\mathrm{CH}_{2} \mathrm{CH}_{2} \mathrm{SCH}_{3}\right)\right) ; \mathrm{m} / z\left(\mathrm{ES}^{+}\right)$ $379\left(\left[{ }^{35} \mathrm{Cl}\right] \mathrm{MH}^{+}\right), 381\left(\left[{ }^{37} \mathrm{Cl}\right] \mathrm{MH}^{+}\right)$; HRMS $\left(\mathrm{ES}^{+}\right)$found $\left[{ }^{35} \mathrm{Cl}\right] \mathrm{MH}^{+}, 379.0994\left(\mathrm{C}_{17} \mathrm{H}_{20} \mathrm{~N}_{4} \mathrm{O}_{2} \mathrm{~S}^{35} \mathrm{Cl}\right.$ requires 379.0995).

(S)-N-(1-Oxo-1-(2-(4-(trifluoromethyl)phenyl)hydrazineyl)propan-2-yl)pyrazine-2-carboxamide, 14, following the standard procedure B, tert-butyl (S)-(1-oxo-1-(2-(4-(trifluoromethyl) phenyl)hydrazineyl)propan-2-yl) carbamate $(0.10 \mathrm{~g}, 0.30 \mathrm{mmol})$ and pyrazinoic acid were transformed following flash chromatography ( $n$-hexane/EtOAc [4:1] to afford the title compound as a white solid (54 mg, 52\%); $\mathrm{R}_{\mathrm{f}} 0.19$ (DCM/EtOH/ sat. aq. $\mathrm{NH}_{3}$ [200:8:1]); $v_{\max } 3255(\mathrm{~N}-\mathrm{H})$, 3098, $2981(\mathrm{C}-\mathrm{H}), 1638(\mathrm{C}=\mathrm{O}), 1617,1534,1326(\mathrm{C}-\mathrm{F})$, $1156(\mathrm{C}-\mathrm{N}), 1101,1065(\mathrm{C}-\mathrm{F}) \mathrm{cm}^{-1} ; \delta_{\mathrm{H}}\left(400 \mathrm{MHz}, \mathrm{DMSO}-\mathrm{d}_{6}\right) 10.10(1 \mathrm{H}, \mathrm{bs}, \mathrm{CONHNH}), 9.22(1 \mathrm{H}$, s, Pyz- $H), 8.91(1 \mathrm{H}, \mathrm{m}$, Pyz- $H), 8.87(1 \mathrm{H}, \mathrm{d}, J$ J, PyzCONH), $8.77(1 \mathrm{H}, \mathrm{d}, J$ 2, Pyz- $H), 8.43(1 \mathrm{H}, \mathrm{bs}$, $\mathrm{CONHNH}), 7.48\left(2 \mathrm{H}, \mathrm{d}, J\right.$ 8, Ar-H), $6.84(2 \mathrm{H}, \mathrm{d}, J$ 8, $\mathrm{Ar}-\mathrm{H}), 4.60\left(1 \mathrm{H}, \mathrm{m}, \mathrm{NHCH}\left(\mathrm{CH}_{3}\right) \mathrm{CO}\right), 1.50(3 \mathrm{H}, \mathrm{d}, J$ 7, NHCH$\left.\left(\mathrm{CH}_{3}\right) \mathrm{CO}\right) ; \delta_{\mathrm{C}}\left(100 \mathrm{MHz}, \mathrm{DMSO}-\mathrm{d}_{6}\right) 172.2$ (CONHNH), 163.1 (PyzCO), 152.8 (ipso-Ar-C), 148.2 (Pyz-C), 144.9 (ipso-Pyz-C), 143.99 (Pyz-C), 143.85 (Pyz-C), 126.6 (Ar-C), 123.3 (1C, q, ${ }^{1} J_{C-F} 269$, $\left.\mathrm{CF}_{3}\right), 118.5\left(1 \mathrm{C}, \mathrm{q},{ }^{2} \mathrm{~J}_{\mathrm{C}-\mathrm{F}} 32\right.$, ipso-Ar- $\left.\mathrm{CCF}_{3}\right), 111.9(\mathrm{Ar}-\mathrm{C}), 47.9\left(\mathrm{NHCH}\left(\mathrm{CH}_{3}\right) \mathrm{CO}\right), 18.7\left(\mathrm{NHCH}\left(\mathrm{CH}_{3}\right) \mathrm{CO}\right)$; $\delta_{\mathrm{F}}\left(282 \mathrm{MHz}, \mathrm{DMSO}-\mathrm{d}_{6}\right)-59.31\left(3 \mathrm{~F}, \mathrm{~s}, \mathrm{CF}_{3}\right) ; \mathrm{m} / z\left(\mathrm{ES}^{+}\right) 354\left(\mathrm{MH}^{+}\right) ; \mathrm{HRMS}\left(\mathrm{ES}^{+}\right)$found $\mathrm{MH}^{+}, 354.1188$ $\left(\mathrm{C}_{15} \mathrm{H}_{15} \mathrm{~F}_{3} \mathrm{~N}_{5} \mathrm{O}_{2}\right.$ requires 354.1178).

(S)-N-(4-(methylthio)-1-oxo-1-(2-(4-(trifluoromethyl)phenyl)hydrazineyl)butan-2-yl)pyrazine-2-carboxamide, 15, following the standard procedure B, tert-Butyl $N-\left[(1 S)-3-\left(\right.\right.$ methylsulfanyl)-1-\{ $N^{\prime}$-[4-(trifluoromethyl) phenyl]hydrazine carbonyl)propyl]carbamate $(78 \mathrm{mg}, 0.23 \mathrm{mmol})$ and pyrazinoic acid were transformed to afford the title compound as a yellow semisolid (80 mg, 86\%); $v_{\max }$ (ATR) 3264, 1661, $1616,1519,1404,1322,1157,1105,1064,1019,838,832,771 \mathrm{~cm}^{-1} ; \delta_{\mathrm{H}}\left(300 \mathrm{MHz}, \mathrm{CDCl}_{3}\right) 9.36(1 \mathrm{H}, \mathrm{s}$, Pyz-H), 9.06 (1H, d, J 8, CONHNH), 8.78 (1H, d, J 2, Pyz-H), 8.53 (1H, bs, Pyz-H), $8.43(1 \mathrm{H}, \mathrm{d}, J$, $\mathrm{CONH}), 7.40\left(1 \mathrm{H}, \mathrm{d}, J\right.$ 8, Ar-H), $6.82\left(1 \mathrm{H}, \mathrm{d}, J\right.$ 8, Ar-H), $4.99\left(1 \mathrm{H}, \mathrm{m}, \mathrm{NHCH}\left(\mathrm{CH}_{2} \mathrm{CH}_{2} \mathrm{SCH}_{3}\right)\right), 2.63(2 \mathrm{H}$, $\left.\mathrm{m}, \mathrm{NHCH}\left(\mathrm{CH}_{2} \mathrm{CH}_{2} \mathrm{SCH}_{3}\right)\right)$, 2.35-2.10 $\left(5 \mathrm{H}, \mathrm{m}, \mathrm{NHCH}\left(\mathrm{CH}_{2} \mathrm{CH}_{2} \mathrm{SCH}_{3}\right) ; \delta_{\mathrm{C}}\left(100 \mathrm{MHz}, \mathrm{CDCl}_{3}\right) 171.2\right.$ (CONHNH), 163.7 (Pyz-CONH), 150.4 (ipso-Ar-C), 147.9 (Pyz-C), 145.0 (ipso-Pyz-C), 144.3 (Pyz-C), 143.4 (ipso-Ar-C), 142.8 (Pyz-C), 126.6 (Ar-C), 122.6 (1C, q, ${ }^{2} J_{\mathrm{C}-\mathrm{F}} 31$, ipso-Ar-CCF 3 ), 112.5 (Ar-C), $50.7\left(\mathrm{NHCH}\left(\mathrm{CH}_{2} \mathrm{CH}_{2} \mathrm{SCH}_{3}\right)\right), 30.94\left(\mathrm{NHCH}\left(\mathrm{CH}_{2} \mathrm{CH}_{2} \mathrm{SCH}_{3}\right)\right), 30.15\left(\mathrm{NHCH}\left(\mathrm{CH}_{2} \mathrm{CH}_{2} \mathrm{SCH}_{3}\right)\right), 15.4$ $\left(\mathrm{NHCH}\left(\mathrm{CH}_{2} \mathrm{CH}_{2} \mathrm{SCH}_{3}\right)\right) ; \mathrm{m} / z\left(\mathrm{ES}^{+}\right) 414\left(\mathrm{MH}^{+}\right) ; \mathrm{HRMS}\left(\mathrm{ES}^{+}\right)$found $\mathrm{MH}^{+}, 414.1207\left(\mathrm{C}^{17} \mathrm{H}^{19} \mathrm{~F}^{3} \mathrm{~N}^{5} \mathrm{O}^{2} \mathrm{~S}\right.$ requires 414.1212).

(S)-N-(1-(2-(3-chlorophenyl)hydrazineyl)-1-oxopropan-2-yl)pyrazine-2-carboxamide, 16, following the standard procedure B, tert-butyl (S)-(1-(2-(3-chlorophenyl)hydrazineyl)-1-oxopropan-2-yl)carbamate $(0.10 \mathrm{~g}, 0.32 \mathrm{mmol})$ and pyrazinoic acid were transformed to afford the title compound as a white solid $(63 \mathrm{mg}, 62 \%)$ as a mixture of rotamers [6:1]; $\mathrm{R}_{\mathrm{f}} 0.21\left(\mathrm{DCM} / \mathrm{EtOH} /\right.$ sat. aq. $\mathrm{NH}_{3}$ [200:8:1]); $v_{\max }$ (ATR) 3383, 3293 (N-H), 3029 (Ar-CH), 2922, 2865 (C-H), 1681, 1659 (C=O) 1596, 1536, 1092, 1018, $886 \mathrm{~cm}^{-1}$; NMR data given for major rotamer $\delta_{\mathrm{H}}\left(400 \mathrm{MHz}, \mathrm{CDCl}_{3}\right) 9.35(1 \mathrm{H}, \mathrm{s}, \mathrm{Pyz}-H), 8.89(1 \mathrm{H}, \mathrm{s}$, CONHNH), $8.76(1 \mathrm{H}, \mathrm{d}, J$ 2, Pyz-H), $8.53(1 \mathrm{H}, \mathrm{m}$, Pyz-H), 8.34 (1H, d, J 8, Pyz-CONH), 7.06 (1H, dd, J 8, $\mathrm{Ar}-\mathrm{H}), 6.79\left(1 \mathrm{H}, \mathrm{d}, J\right.$ 8, Ar-H), $6.76(1 \mathrm{H}, \mathrm{s}, \mathrm{Ar}-\mathrm{H}), 6.65(1 \mathrm{H}, \mathrm{d}, J$ 8, $\mathrm{Ar}-\mathrm{H}), 4.85\left(1 \mathrm{H}, \mathrm{m}, \mathrm{NHCH}\left(\mathrm{CH}_{3}\right) \mathrm{CO}\right)$, $1.57\left(3 \mathrm{H}, \mathrm{d}, J \mathrm{~J}, \mathrm{NHCH}\left(\mathrm{CH}_{3}\right) \mathrm{CO}\right) ; \delta_{\mathrm{C}}\left(100 \mathrm{MHz}, \mathrm{CDCl}_{3}\right)$ 172.1 (CONHNH), 163.5 (Pyz-CONH), 149.0 (ipso-Ar-C), 147.8 (Pyz-C), 144.3 (Pyz-C), 143.6 (ipso-Pyz-C), 142.8 (Pyz-C), 135.0 (ipso-Ar-C), 130.2 $(\mathrm{Ar}-\mathrm{C}), 121.1(\mathrm{Ar}-\mathrm{C}), 113.4(\mathrm{Ar}-\mathrm{C}), 111.7(\mathrm{Ar}-\mathrm{C}), 47.58\left(\mathrm{NHCH}\left(\mathrm{CH}_{3}\right) \mathrm{CO}\right), 17.7\left(\mathrm{NHCH}\left(\mathrm{CH}_{3}\right) \mathrm{CO}\right) ; \mathrm{m} / \mathrm{z}$ $\left(\mathrm{ES}^{+}\right) 320\left(\left[{ }^{35} \mathrm{Cl}\right] \mathrm{MH}^{+}\right), 322\left(\left[{ }^{37} \mathrm{Cl}\right] \mathrm{MH}^{+}\right)$; HRMS $\left(\mathrm{ES}^{+}\right)$found $\left[{ }^{35} \mathrm{Cl} \mathrm{MH}^{+}, 320.0914\left(\mathrm{C}_{14} \mathrm{H}_{15} \mathrm{~N}_{5} \mathrm{O}_{2}{ }^{35} \mathrm{Cl}\right.\right.$ requires 320.0914).

(S)-N-(1-(2-(3-chlorophenyl)hydrazineyl)-4-(methylthio)-1-oxobutan-2-yl)pyrazine-2-carboxamide, 17, following the standard procedure B, tert-Butyl $N-\left[(1 S)-1-\left[N^{\prime}\right.\right.$-(3-chlorophenyl)hydrazinecarbonyl]-3(methylsulfanyl)propyl] carbamate $(70 \mathrm{mg}, 0.23 \mathrm{mmol}$ ) and pyrazinoic acid were transformed to 
afford the title compound as a white solid (61 mg, 71\%); m.p. 99-102 ${ }^{\circ} \mathrm{C} ; v_{\max }$ (ATR) 3333, 3290, 2921, 1708, 1678, 1652, 1596, 1585, 1523, 1482, 1438, 1425, 1404, 1375, 1318, 1299, 1272, 1234, $1221,1188,1163,1114,1091,1071,1052,1023,991,958,946,914,864,809 \mathrm{~cm}^{-1} ; \delta_{\mathrm{H}}(400 \mathrm{MHz}$, DMSO-d $\left._{6}\right) 10.03(1 \mathrm{H}, \mathrm{bs}, \mathrm{CONHNH}), 9.22(1 \mathrm{H}, \mathrm{s}$, Pyz- $H), 9.00(1 \mathrm{H}, \mathrm{d}, J$ 8, CONH $),(1 \mathrm{H}, \mathrm{s}$, CONHNH), $8.91(1 \mathrm{H}, \mathrm{d}, J$ 2, Pyz-H), $8.78(1 \mathrm{H}, \mathrm{bs}, \mathrm{Pyz}-H), 8.10(1 \mathrm{H}, \mathrm{bs}, \mathrm{CONHNH}), 7.16(1 \mathrm{H}$, $\mathrm{dd}, J$ 8, Ar-H), 6.74-6.62 (3H, m, Ar-H), $4.70\left(1 \mathrm{H}, \mathrm{m}, \mathrm{NHCH}\left(\mathrm{CH}_{2} \mathrm{CH}_{2} \mathrm{SCH}_{3}\right)\right), 2.59-2.50(2 \mathrm{H}$, $\left.\mathrm{m}, \mathrm{NHCH}\left(\mathrm{CH}_{2} \mathrm{CH}_{2} \mathrm{SCH}_{3}\right)\right)$, 2.18-2.03 (5H, m, NHCH $\left(\mathrm{CH}_{2} \mathrm{CH}_{2} \mathrm{SCH}_{3}\right) ; \delta_{\mathrm{C}}\left(75 \mathrm{MHz}, \mathrm{DMSO}-\mathrm{d}_{6}\right)$ 171.2 (CONHNH), 163.7 (Pyz-CONH), 151.3 (ipso-Ar-C), 148.1 (Pyz-C), 145.0 (ipso-Pyz-C), 144.1 (Pyz-C), 143.8 (Pyz-C), 134.0 (ipso-Ar-C), 130.8 (Ar-C), 118.4 (Ar-C), 111.9 (Ar-C), 111.3 (Ar-C), $51.7\left(\mathrm{NHCH}\left(\mathrm{CH}_{2} \mathrm{CH}_{2} \mathrm{SCH}_{3}\right)\right)$, $31.6\left(\mathrm{NHCH}\left(\mathrm{CH}_{2} \mathrm{CH}_{2} \mathrm{SCH}_{3}\right)\right), 30.2\left(\mathrm{NHCH}\left(\mathrm{CH}_{2} \mathrm{CH}_{2} \mathrm{SCH}_{3}\right)\right), 15.1$ $\left(\mathrm{NHCH}\left(\mathrm{CH}_{2} \mathrm{CH}_{2} \mathrm{SCH}_{3}\right)\right) ; \mathrm{m} / z\left(\mathrm{ES}^{+}\right) 380\left(\left[{ }^{35} \mathrm{Cl}\right] \mathrm{MH}^{+}\right), 382\left(\left[{ }^{37} \mathrm{Cl}\right] \mathrm{MH}^{+}\right) ; \mathrm{HRMS}\left(\mathrm{ES}^{+}\right)$found $\left[{ }^{35} \mathrm{Cl}\right] \mathrm{MH}^{+}$, $380.0948\left(\mathrm{C}_{16} \mathrm{H}_{19}{ }^{35} \mathrm{ClN}_{5} \mathrm{O}_{2} \mathrm{~S}\right.$ requires 380.0948).

(S)-N-(1-Oxo-1-(2-(4-(trifluoromethyl)phenyl)hydrazineyl)propan-2-yl)-2-naphthamide, 18, following the standard procedure B, tert-butyl (S)-(1-oxo-1-(2-(4-(trifluoromethyl) phenyl)hydrazineyl)propan-2-yl) carbamate $(0.10 \mathrm{~g}, 0.29 \mathrm{mmol})$ and 2-napthoic acid were transformed to afford the title compound as a white solid (65 mg, 56\%); $\mathrm{R}_{\mathrm{f}} 0.24\left(\mathrm{DCM} / \mathrm{EtOH} / \mathrm{sat}\right.$. aq. $\mathrm{NH}_{3}$ [200:8:1]); $v_{\max }(\mathrm{ATR}) 3329,3264(\mathrm{~N}-\mathrm{H})$, 3051, 2995, 2854, 1674, 1618 (C=O), 1525, 1325 (C-F), $1160(\mathrm{C}-\mathrm{N}), 1095,1065$ (C-F), $834(\mathrm{C}-\mathrm{C}) \mathrm{cm}^{-1}$; $\delta_{\mathrm{H}}$ (400 MHz, DMSO) $10.04(1 \mathrm{H}, \mathrm{bs}, \mathrm{CONHNH}), 8.82(1 \mathrm{H}, \mathrm{d}, J$ 2, Np-CONH), $8.55(1 \mathrm{H}, \mathrm{s}, \mathrm{Np}-\mathrm{H}), 8.42(1 \mathrm{H}$, d, J 2, CONHNH), 8.08-7.97 (4H, m, Np-H), 7.66-7.58 (2H, m, Np-H), 7.47 (2H, d, J 8, Ar-H), 6.87 $(2 \mathrm{H}, \mathrm{d}, J 8, \mathrm{Ar}-\mathrm{H}), 4.60\left(1 \mathrm{H}, \mathrm{m}, \mathrm{NHCH}\left(\mathrm{CH}_{3}\right)\right), 1.49\left(3 \mathrm{H}, \mathrm{d}, J 7, \mathrm{NHCH}\left(\mathrm{CH}_{3}\right)\right) ; \delta_{\mathrm{C}}\left(176 \mathrm{MHz}, \mathrm{DMSO}-\mathrm{d}_{6}\right)$ 173.1 (CONHNH), 166.9 (NpCONH), 153.0 (ipso-Ar-C), 134.7 (ipso-Ar-C), 132.5 (ipso-Ar-C), 131.7 (ipso-Ar-C), $129.3(\mathrm{~Np}-\mathrm{C}), 128.4(\mathrm{~Np}-\mathrm{C}), 128.4(\mathrm{~Np}-\mathrm{C}), 128.2(\mathrm{Ar}-\mathrm{C}), 128.1(\mathrm{~Np}-\mathrm{C}), 127.2(\mathrm{~Np}-\mathrm{C}), 126.6$ $(\mathrm{Np}-\mathrm{C}), 125.0(\mathrm{~Np}-\mathrm{C}), 123.7\left(1 \mathrm{C}, \mathrm{q},{ }^{1} J_{\mathrm{C}-\mathrm{F}} 269, \mathrm{CF}_{3}\right), 118.6\left(1 \mathrm{C}, \mathrm{q},{ }^{2} J_{\mathrm{C}-\mathrm{F}} 31, i p s o-\mathrm{Ar}^{-C_{C}} \mathrm{~F}_{3}\right), 112.0(\mathrm{Ar}-\mathrm{C})$, $48.7\left(\mathrm{NHCH}\left(\mathrm{CH}_{3}\right)\right), 18.2\left(\mathrm{NHCH}\left(\mathrm{CH}_{3}\right)\right) ; \delta_{\mathrm{F}}\left(282 \mathrm{MHz}, \mathrm{DMSO}-\mathrm{d}_{6}\right)-59.3\left(3 \mathrm{~F}, \mathrm{~s}, \mathrm{CF}_{3}\right) ; m / z\left(\mathrm{ES}^{+}\right) 402$ $\left(\mathrm{MH}^{+}\right)$; HRMS $\left(\mathrm{ES}^{+}\right)$found $\mathrm{MH}^{+}, 402.1427\left(\mathrm{C}_{21} \mathrm{H}_{19} \mathrm{~F}_{3} \mathrm{~N}_{3} \mathrm{O}_{2}\right.$ requires 402.1429).

(S)-N-(4-(methylthio)-1-oxo-1-(2-(4-(trifluoromethyl)phenyl)hydrazineyl)butan-2-yl)-2-naphthamide, 19, following the standard procedure B, tert-Butyl $N$-[(1S)-3-(methylsulfanyl)-1-\{ $N^{\prime}$-[4-(trifluoromethyl) phenyl]hydrazine carbonyl\}propyl]carbamate $(65 \mathrm{mg}, 0.19 \mathrm{mmol})$ and 2-naphthoic acid were transformed to afford the title compound as a white solid (53 mg, 61\%); m.p. $163-166^{\circ} \mathrm{C} ; v_{\max }$ (ATR) $3264,1666,1635,1615,1520,1503,1432,1325,1232,1159,1103,1065,1011,955,897,865,838 \mathrm{~cm}^{-1} ; \delta_{\mathrm{H}}$ (300 MHz, DMSO-d 6 ) $10.12(1 \mathrm{H}, \mathrm{bs}, \mathrm{CONHNH}), 8.83(1 \mathrm{H}, \mathrm{d}, J$ 7, Np-CONH), $8.56(1 \mathrm{H}, \mathrm{s}, \mathrm{Np}-H), 8.44$ (1H, bs, CONHNH), 8.10-7.94 (4H, m, Np-H), 7.65-7.55 (2H, m, Np-H), $7.60(2 \mathrm{H}, \mathrm{d}, J$ 8, Ar-H), 6.87 $(1 \mathrm{H}, \mathrm{d}, \mathrm{J} 8, \mathrm{Ar}-\mathrm{H}), 4.69\left(1 \mathrm{H}, \mathrm{m}, \mathrm{NHCH}\left(\mathrm{CH}_{2} \mathrm{CH}_{2} \mathrm{SCH}_{3}\right)\right), 2.74-2.55\left(2 \mathrm{H}, \mathrm{m}, \mathrm{NHCH}\left(\mathrm{CH}_{2} \mathrm{CH}_{2} \mathrm{SCH}_{3}\right)\right)$, 2.19-2.06 (5H, m, NHCH $\left(\mathrm{CH}_{2} \mathrm{CH}_{2} \mathrm{SCH}_{3}\right) ; \delta_{\mathrm{C}}\left(75 \mathrm{MHz}, \mathrm{DMSO}_{6}\right)$ 172.1 (CONHNH), $167.4(\mathrm{CONH})$, 152.9 (ipso-Ar-C), 134.7 (ipso-Ar-C), 134.0 (ipso-Ar-C), 132.5 (ipso-Ar-C), 131.8 (ipso-Ar-C), 129.3 $(\mathrm{Np}-\mathrm{C}), 128.4(\mathrm{~Np}-\mathrm{C}), 128.2(\mathrm{~Np}-\mathrm{C}), 128.1(\mathrm{~Np}-\mathrm{C}), 127.2(\mathrm{~Np}-\mathrm{C}), 126.6(\mathrm{Ar}-\mathrm{C}), 125.0(\mathrm{~Np}-\mathrm{C}), 118.8(1 \mathrm{C}$, q, ${ }^{2} J_{\mathrm{C}-\mathrm{F}} 32$, ipso-Ar- $\left.\mathrm{CCF}_{3}\right), 111.9$ (Ar-C), $52.3\left(\mathrm{NHCH}\left(\mathrm{CH}_{2} \mathrm{CH}_{2} \mathrm{SCH}_{3}\right)\right), 31.5\left(\mathrm{NHCH}\left(\mathrm{CH}_{2} \mathrm{CH}_{2} \mathrm{SCH}_{3}\right)\right)$, $30.5\left(\mathrm{NHCH}\left(\mathrm{CH}_{2} \mathrm{CH}_{2} \mathrm{SCH}_{3}\right)\right), 15.2\left(\mathrm{NHCH}\left(\mathrm{CH}_{2} \mathrm{CH}_{2} \mathrm{SCH}_{3}\right)\right) ; \delta_{\mathrm{F}}\left(282 \mathrm{MHz}, \mathrm{DMSO}-\mathrm{d}_{6}\right) 59.26(3 \mathrm{~F}$, $\left.\mathrm{s}, \mathrm{CF}_{3}\right) ; \mathrm{m} / z\left(\mathrm{ES}^{+}\right) 462\left(\mathrm{MH}^{+}\right), 484\left(\mathrm{MNa}^{+}\right) ; \mathrm{HRMS}\left(\mathrm{ES}^{+}\right)$found $\mathrm{MH}^{+}, 462.1465\left(\mathrm{C}_{23} \mathrm{H}_{23} \mathrm{~F}_{3} \mathrm{~N}_{3} \mathrm{O}_{2} \mathrm{~S}\right.$ requires 462.1463$)$.

(S)-N-(1-(2-(3-chlorophenyl)hydrazineyl)-1-oxopropan-2-yl)-2-naphthamide, 20, following the standard procedure B, tert-butyl (S)-(1-(2-(3-chlorophenyl)hydrazineyl)-1-oxopropan-2-yl)carbamate (0.10 g, $0.32 \mathrm{mmol}$ ) and 2-napthoic acid were transformed to afford the title compound as a white solid (86 $\mathrm{mg}, 73 \%) ; \mathrm{R}_{\mathrm{f}} 0.32$ (DCM/EtOH/ sat. aq. $\mathrm{NH}_{3}$ [200:8:1]); $v_{\max }(\mathrm{ATR}) 3331,3236(\mathrm{NH}), 3062(\mathrm{Ar}-\mathrm{CH})$, 2983, $2926(\mathrm{CH}), 1634,1626(\mathrm{C}=\mathrm{O}), 1599,1536,1536(\mathrm{C}=\mathrm{C}), 1334(\mathrm{C}-\mathrm{N}) \mathrm{cm}^{-1} ; \delta_{\mathrm{H}}\left(400 \mathrm{MHz}, \mathrm{DMSO}-\mathrm{d}_{6}\right)$ $9.96(1 \mathrm{H}, \mathrm{bs}, \mathrm{CONHNH}), 8.82(1 \mathrm{H}, \mathrm{d}, J$ 7, Np-CONH), $8.56(1 \mathrm{H}, \mathrm{s}, \mathrm{Np}-H), 8.07-7.95(4 \mathrm{H}, \mathrm{m}, \mathrm{Np}-H)$, 7.67-7.57 (2H, m, Np-H), $7.14(1 \mathrm{H}, \mathrm{dd}, J$ 8, Ar-H), $6.77(1 \mathrm{H}, \mathrm{t}, J \mathrm{2}, \mathrm{Ar}-\mathrm{H}), 6.73-6.69(2 \mathrm{H}, \mathrm{m}, \mathrm{Ar}-\mathrm{H})$, $4.58\left(1 \mathrm{H}, \mathrm{m}, \mathrm{NHCH}\left(\mathrm{CH}_{3}\right) \mathrm{CO}\right), 1.47\left(3 \mathrm{H}, \mathrm{d}, J\right.$ 7, $\left.\mathrm{NHCH}\left(\mathrm{CH}_{3}\right) \mathrm{CO}\right) ; \delta_{\mathrm{C}}\left(100 \mathrm{MHz}, \mathrm{DMSO}_{6} \mathrm{~d}_{6}\right) 173.0$ 
(CHCONHNH), 166.9 (Np-CONH), 151.4 (Ar-C), 134.7 (Np-C), 134.0 (ipso-Ar-C), 132.5 (Np-C), 131.8 $(\mathrm{Np}-\mathrm{C}), 130.8(\mathrm{Ar}-\mathrm{C}), 129.3(\mathrm{~Np}-\mathrm{C}), 128.3(\mathrm{~Np}-\mathrm{C}), 128.2(\mathrm{~Np}-\mathrm{C}), 128.1(\mathrm{~Np}-\mathrm{C}), 127.2(\mathrm{~Np}-\mathrm{C}), 125.0$ $(\mathrm{Np}-\mathrm{C}), 118.2(\mathrm{Ar}-\mathrm{C}), 111.9(\mathrm{Ar}-\mathrm{C}), 111.3(\mathrm{Ar}-\mathrm{C}), 48.7\left(\mathrm{NHCH}\left(\mathrm{CH}_{3}\right) \mathrm{CO}\right), 18.1\left(\mathrm{NHCH}\left(\mathrm{CH}_{3}\right) \mathrm{CO}\right) ; \mathrm{m} / \mathrm{z}$ $\left.\left.\left(\mathrm{ES}^{+}\right) 368\left({ }^{[35} \mathrm{Cl}\right] \mathrm{MH}^{+}\right), 370\left({ }^{[37} \mathrm{Cl}\right] \mathrm{MH}^{+}\right)$; HRMS $\left(\mathrm{ES}^{+}\right)$found $\left(\left[{ }^{35} \mathrm{Cl}^{-} \mathrm{MH}^{+}, 368.1170\left(\mathrm{C}_{20} \mathrm{H}_{19} \mathrm{~N}_{3} \mathrm{O}_{2}{ }^{35} \mathrm{Cl}\right.\right.\right.$ requires 368.1166$)$.

(S)-N-(1-(2-(3-chlorophenyl)hydrazineyl)-4-(methylthio)-1-oxobutan-2-yl)-2-naphthamide, 21, following the standard procedure B, tert-Butyl N-[(1S)-1-[N'-(3-chlorophenyl)hydrazinecarbonyl]-3-(methylsulfanyl) propyl] carbamate $(70 \mathrm{mg}, 0.23 \mathrm{mmol})$ and 2-naphthoic acid were transformed to afford the title compound as a white solid (18 mg, 19\%); m.p. 205-208 ${ }^{\circ} \mathrm{C}$; $v_{\max }$ (ATR) $3224,1636,1625,1597,1527,1494$, $1452,1440,1335,1303,1268,1231,1201,1076,989,955,892,863,839 \mathrm{~cm}^{-1} ; \delta_{\mathrm{H}}(400 \mathrm{MHz}$, DMSO-d 6$) 10.04$ (1H, bs, CONHNH), 8.85 (1H, d, J 7, Np-CONH), $8.56(1 \mathrm{H}, \mathrm{s}, \mathrm{Np}-H), 8.11(1 \mathrm{H}, \mathrm{bs}, \mathrm{CONHNH}), 8.10-7.97$ (4H, m, Np-H), 7.68-7.54 (2H, m, Np-H), 7.15 (1H, dd, J 8, Ar-H), 6.77 (1H, t, J 2, Ar-H), 6.73-6.65 (2H, $\mathrm{m}, \mathrm{Ar}-\mathrm{H}), 4.67\left(1 \mathrm{H}, \mathrm{m}, \mathrm{NHCH}\left(\mathrm{CH}_{2} \mathrm{CH}_{2} \mathrm{SCH}_{3}\right)\right), 2.68-2.56\left(2 \mathrm{H}, \mathrm{m}, \mathrm{NHCH}\left(\mathrm{CH}_{2} \mathrm{CH}_{2} \mathrm{SCH}_{3}\right)\right), 2.12-2.06$ $\left(5 \mathrm{H}, \mathrm{m}, \mathrm{NHCH}\left(\mathrm{CH}_{2} \mathrm{CH}_{2} \mathrm{SCH}_{3}\right) ; \delta_{\mathrm{C}}\left(176 \mathrm{MHz}, \mathrm{DMSO}_{-} \mathrm{d}_{6}\right) 172.0(\mathrm{CHCONHNH}), 167.4(\mathrm{~Np}-\mathrm{CONH})\right.$, 151.4 (Ar-C), 134.7 ( Np-C), 134.0 (ipso-Ar-C), $132.5(\mathrm{~Np}-\mathrm{C}), 131.8$ (Np-C), 130.8 (Ar-C), 129.3 (Np-C), $128.3(\mathrm{~Np}-\mathrm{C}), 128.2(\mathrm{~Np}-\mathrm{C}), 128.1(\mathrm{~Np}-\mathrm{C}), 127.7(\mathrm{~Np}-\mathrm{C}), 125.0(\mathrm{~Np}-\mathrm{C}), 118.3(\mathrm{Ar}-\mathrm{C}), 111.9(\mathrm{Ar}-\mathrm{C}), 111.3$ (Ar-C), $52.3\left(\mathrm{NHCH}\left(\mathrm{CH}_{2} \mathrm{CH}_{2} \mathrm{SCH}_{3}\right)\right), 31.4\left(\mathrm{NHCH}\left(\mathrm{CH}_{2} \mathrm{CH}_{2} \mathrm{SCH}_{3}\right)\right), 30.5\left(\mathrm{NHCH}\left(\mathrm{CH}_{2} \mathrm{CH}_{2} \mathrm{SCH}_{3}\right)\right), 15.1$ $\left(\mathrm{NHCH}\left(\mathrm{CH}_{2} \mathrm{CH}_{2} \mathrm{SCH}_{3}\right)\right) ; \mathrm{m} / z\left(\mathrm{ES}^{+}\right) 428\left(\left[{ }^{35} \mathrm{Cl} \mathrm{MH}^{+}\right), 430\left(\left[{ }^{37} \mathrm{Cl}\right] \mathrm{MH}^{+}\right) ; \mathrm{HRMS}\left(\mathrm{ES}^{+}\right)\right.$found $\left[{ }^{35} \mathrm{Cl}\right] \mathrm{MH}^{+}$, $428.1201\left(\mathrm{C}_{22} \mathrm{H}_{23}{ }^{35} \mathrm{ClN}_{3} \mathrm{O}_{2} \mathrm{~S}\right.$ requires 428.1200$)$.

(S)-N-(1-Oxo-1-(2-(4-(trifluoromethyl)phenyl)hydrazineyl)propan-2-yl)-1-naphthamide,

22, following the standard procedure B, tert-butyl (S)-(1-oxo-1-(2-(4-(trifluoromethyl) phenyl)hydrazineyl)propan-2-yl)carbamate $(0.10 \mathrm{~g}, 0.29 \mathrm{mmol})$ and 1-napthoic acid were transformed to afford the title compound as a white solid (54 mg, 46\%); $\mathrm{R}_{\mathrm{f}} 0.24(\mathrm{DCM} / \mathrm{EtOH} / \mathrm{sat}$. aq. $\mathrm{NH}_{3}$ [200:8:1]; $v_{\max }(\mathrm{ATR})$ 3315, $3242(\mathrm{~N}-\mathrm{H}), 3074(\mathrm{C}-\mathrm{H}), 2999,2865(\mathrm{C}-\mathrm{H}), 1667,1633(\mathrm{C}=\mathrm{O}), 1617$, 1536, $1324(\mathrm{C}-\mathrm{F}), 1159(\mathrm{C}-\mathrm{N})$, 1095, 1065 (C-F), 828, 787, $776(\mathrm{C}-\mathrm{C}) \mathrm{cm}^{-1} ; \delta_{\mathrm{H}}\left(400 \mathrm{MHz}, \mathrm{DMSO}-\mathrm{d}_{6}\right) 10.10$ $(1 \mathrm{H}, \mathrm{bs}, \mathrm{CONHNH}), 8.83(1 \mathrm{H}, \mathrm{d}, J$ 7, NpCONH$), 8.47(1 \mathrm{H}, \mathrm{bs}, \mathrm{CONHNH}), 8.28(1 \mathrm{H}, \mathrm{d}, J$,, $\mathrm{Np}-H), 8.03$ $(1 \mathrm{H}, \mathrm{d}, J$ 8, Np-H), $7.98(1 \mathrm{H}, \mathrm{d}, J$ 8, Np-H) $7.68(1 \mathrm{H}, \mathrm{d}, J$ 7, Np-H), 7.59-7.50 (3H, m, Np-H), $7.47(2 \mathrm{H}, \mathrm{d}$, $J$ 8, $\mathrm{Ar}-\mathrm{H}), 6.91(2 \mathrm{H}, \mathrm{d}, J$ 8, $\mathrm{Ar}-\mathrm{H}), 4.60\left(1 \mathrm{H}, \mathrm{m}, \mathrm{NHCH}\left(\mathrm{CH}_{3}\right) \mathrm{CO}\right), 1.44\left(3 \mathrm{H}, \mathrm{d}, J 7, \mathrm{NHCH}\left(\mathrm{CH}_{3}\right) \mathrm{CO}\right) ; \delta_{\mathrm{C}}$ (100 MHz, DMSO-d 6 ) 173.1 (CONHNH), 167.3 (NpCONH), 153.0 (ipso-NHNHAr-C), 134.1 (Np-C), $134.0(\mathrm{~Np}-\mathrm{C}), 133.9(\mathrm{~Np}-\mathrm{C}), 130.2(\mathrm{~Np}-\mathrm{C}), 128.6(\mathrm{~Np}-\mathrm{C}), 126.9(\mathrm{~Np}-\mathrm{C}), 126.7(\mathrm{~Np}-\mathrm{C}), 126.6(\mathrm{Ar}-\mathrm{C})$, $126.1(\mathrm{~Np}-\mathrm{C}), 125.9(\mathrm{~Np}-\mathrm{C}), 125.3(\mathrm{~Np}-\mathrm{C}), 118.4\left(1 \mathrm{C}, \mathrm{q},{ }^{2} J_{\mathrm{C}-\mathrm{F}} 32, i p s o-\mathrm{Ar}_{-} \mathrm{CCF}_{3}\right), 112.0(\mathrm{Ar}-\mathrm{C}), 48.5$ $\left(\mathrm{NHCH}\left(\mathrm{CH}_{3}\right) \mathrm{CO}\right), 18.0\left(\mathrm{NHCH}\left(\mathrm{CH}_{3}\right) \mathrm{CO}\right) ; \delta_{\mathrm{F}}\left(282 \mathrm{MHz}, \mathrm{DMSO}-\mathrm{d}_{6}\right)-59.25\left(3 \mathrm{~F}, \mathrm{~s}, \mathrm{CF}_{3}\right) ; \mathrm{m} / z\left(\mathrm{ES}^{+}\right) 402$ $\left(\mathrm{MH}^{+}\right)$; HRMS $\left(\mathrm{ES}^{+}\right)$found $\mathrm{MH}^{+}, 402.1433\left(\mathrm{C}_{21} \mathrm{H}_{19} \mathrm{~F}_{3} \mathrm{~N}_{3} \mathrm{O}_{2}\right.$ requires 402.1429).

(S)-N-(4-(methylthio)-1-oxo-1-(2-(4-(trifluoromethyl)phenyl)hydrazineyl)butan-2-yl)-1-naphthamide, 23, following the standard procedure B, tert-Butyl $N-\left[(1 S)-3-(m e t h y l s u l f a n y l)-1-\left\{N^{\prime}\right.\right.$-[4-(trifluoromethyl) phenyl]hydrazine carbonyl\}propyl]carbamate $(65 \mathrm{mg}, 0.19 \mathrm{mmol})$ and 1-naphthoic acid were transformed to afford the title compound as a white solid (53 mg, 61\%); m.p. $189-192{ }^{\circ} \mathrm{C} ; v_{\max }$ (ATR) 3239, 1671, 1635, 1616, 1592, 1521, 1439, 1414, 1378, 1325, 1256, 1231, 1182, 1104, 1065, 1011, 966, 939, 886, $834 \mathrm{~cm}^{-1} ; \delta_{\mathrm{H}}\left(300 \mathrm{MHz}, \mathrm{DMSO}-\mathrm{d}_{6}\right) 10.18(1 \mathrm{H}, \mathrm{bs}, \mathrm{CONHNH}), 8.86(1 \mathrm{H}, \mathrm{d}, J$, $\mathrm{NpCONH}), 8.50(1 \mathrm{H}, \mathrm{bs}, \mathrm{CONHNH}), 8.27(1 \mathrm{H}, \mathrm{d}, J$ 8, Np-H), 8.08-7.94 (2H, m, Np-H), 7.66-7.52 $(3 \mathrm{H}, \mathrm{m}, \mathrm{Np}-\mathrm{H}), 7.47\left(2 \mathrm{H}, \mathrm{d}, J\right.$ 8, Ar-H), $6.92(2 \mathrm{H}, \mathrm{d}, J$ 8, $\mathrm{Ar}-\mathrm{H}), 4.70\left(1 \mathrm{H}, \mathrm{m}, \mathrm{NHCH}\left(\mathrm{CH}_{2} \mathrm{CH}_{2} \mathrm{SCH}_{3}\right)\right)$, 2.74-2.55 (2H, m, NHCH $\left.\left(\mathrm{CH}_{2} \mathrm{CH}_{2} \mathrm{SCH}_{3}\right)\right)$, 2.15-2.01 (5H, m, $\mathrm{NHCH}\left(\mathrm{CH}_{2} \mathrm{CH}_{2} \mathrm{SCH}_{3}\right) ; \delta_{\mathrm{C}}(75 \mathrm{MHz}$, DMSO-d $_{6}$ ) 172.1 (CONHNH), 167.3 (NpCONH), 153.0 (ipso-Ar-C), 134.7 (ipso-Ar-C), 133.6 (ipso-Ar-C), 130.4 (ipso-Ar-C), 130.3 (Np-C), $128.6(\mathrm{~Np}-\mathrm{C}), 127.0(\mathrm{~Np}-\mathrm{C}), 126.7$ (Ar-C), 126.5 (ipso-Ar-C), 126.1 $(\mathrm{Np}-\mathrm{C}), 126.0(\mathrm{~Np}-\mathrm{C}), 125.4(\mathrm{~Np}-\mathrm{C}), 125.4(\mathrm{~Np}-\mathrm{C}), 118.3\left(1 \mathrm{C}, \mathrm{q},{ }^{2} \mathrm{~J}_{\mathrm{C}-\mathrm{F}} 31, i p s o-\mathrm{Ar}-\mathrm{CCF}_{3}\right), 112.0(\mathrm{Ar}-\mathrm{C})$, $52.1\left(\mathrm{NHCH}\left(\mathrm{CH}_{2} \mathrm{CH}_{2} \mathrm{SCH}_{3}\right)\right)$, $31.4\left(\mathrm{NHCH}\left(\mathrm{CH}_{2} \mathrm{CH}_{2} \mathrm{SCH}_{3}\right)\right), 30.4\left(\mathrm{NHCH}\left(\mathrm{CH}_{2} \mathrm{CH}_{2} \mathrm{SCH}_{3}\right)\right), 15.1$ $\left(\mathrm{NHCH}\left(\mathrm{CH}_{2} \mathrm{CH}_{2} \mathrm{SCH}_{3}\right)\right) ; \delta_{\mathrm{F}}\left(282 \mathrm{MHz}, \mathrm{DMSO}-\mathrm{d}_{6}\right) 59.27\left(3 \mathrm{~F}, \mathrm{~s}, \mathrm{CF}_{3}\right) ; \mathrm{m} / z\left(\mathrm{ES}^{+}\right) 462\left(\mathrm{MH}^{+}\right), 484\left(\mathrm{MNa}^{+}\right)$; HRMS $\left(\mathrm{ES}^{+}\right)$found $\mathrm{MH}^{+}, 462.1468\left(\mathrm{C}_{23} \mathrm{H}_{23} \mathrm{~F}_{3} \mathrm{~N}_{3} \mathrm{O}_{2} \mathrm{~S}\right.$ requires 462.1463). 
(S)-N-(1-(2-(3-chlorophenyl)hydrazineyl)-1-oxopropan-2-yl)-1-naphthamide, 24, following the standard procedure B, tert-butyl (S)-(1-(2-(3-chlorophenyl)hydrazineyl)-1-oxopropan-2-yl)carbamate (0.10 g, $0.32 \mathrm{mmol}$ ) and 1-napthoic acid were transformed to afford the title compound as a white solid (55 $\mathrm{mg}$, 45\%); $v_{\max }(\mathrm{ATR})$ 3404, $3237(\mathrm{NH}), 3052(\mathrm{Ar}-\mathrm{CH}), 2995,2946(\mathrm{CH}), 1634(\mathrm{C}=\mathrm{O}), 1595(\mathrm{C}=\mathrm{C}), 1324(\mathrm{C}-\mathrm{N})$ $\mathrm{cm}^{-1} ; \delta_{\mathrm{H}}\left(400 \mathrm{MHz}, \mathrm{DMSO}_{6}\right) 10.00(1 \mathrm{H}, \mathrm{bs}, \mathrm{CONHNH}), 8.83(1 \mathrm{H}, \mathrm{d}, J 7, \mathrm{~Np}-\mathrm{CONH}), 8.32(1 \mathrm{H}, \mathrm{dd}$, $J ~ 8,2, \mathrm{~Np}-H), 8.15(1 \mathrm{H}, \mathrm{s}, \mathrm{CONHNH}), 8.03(1 \mathrm{H}, \mathrm{d}, J$ 8, Np-H), $7.98(1 \mathrm{H}, \mathrm{dd}, J$ 8, 2, Np-H), $7.68(1 \mathrm{H}$, $\mathrm{d}, J$ 8, Np-H), 7.60-7.50 (2H, m, Np-H), $7.16(1 \mathrm{H}, \mathrm{dd}, J$ 8, Ar-H), $6.83(1 \mathrm{H}, \mathrm{bs}, \mathrm{Ar}-\mathrm{H}), 6.76-6.71(2 \mathrm{H}$, $\mathrm{m}, \mathrm{Ar}-\mathrm{H}), 4.59\left(1 \mathrm{H}, \mathrm{m}, \mathrm{NHCH}\left(\mathrm{CH}_{3}\right) \mathrm{CO}\right), 1.42\left(3 \mathrm{H}, \mathrm{d}, \mathrm{J} 7, \mathrm{NHCH}\left(\mathrm{CH}_{3}\right) \mathrm{CO}\right) ; \delta_{\mathrm{C}}\left(100 \mathrm{MHz}, \mathrm{DMSO}-\mathrm{d}_{6}\right)$ 173.1 (CONHNH), 169.2 (Np-CONH), $151.4(\mathrm{Ar}-\mathrm{C}), 134.6$ (Np-C), $134.0(\mathrm{Ar}-\mathrm{C}), 133.5(\mathrm{~Np}-\mathrm{C}), 130.8$ $(\mathrm{Ar}-\mathrm{C}), 130.3(\mathrm{~Np}-\mathrm{C}), 128.5(\mathrm{~Np}-\mathrm{C}), 127.0(\mathrm{~Np}-\mathrm{C}), 126.6(\mathrm{~Np}-\mathrm{C}), 126.2(\mathrm{~Np}-\mathrm{C}), 126.0(\mathrm{~Np}-\mathrm{C}), 125.3$ $(\mathrm{Np}-\mathrm{C}), 118.3(\mathrm{Ar}-\mathrm{C}), 111.9(\mathrm{Ar}-\mathrm{C}), 111.3(\mathrm{Ar}-\mathrm{C}), 48.4\left(\mathrm{NHCH}\left(\mathrm{CH}_{3}\right) \mathrm{CO}\right), 18.0\left(\mathrm{NHCH}\left(\mathrm{CH}_{3}\right) \mathrm{CO}\right) ; \mathrm{m} / \mathrm{z}$ $\left.\left.\left(\mathrm{ES}^{+}\right) 368\left({ }^{[35} \mathrm{Cl}\right] \mathrm{MH}^{+}\right), 370\left({ }^{[37} \mathrm{Cl}\right] \mathrm{MH}^{+}\right)$; HRMS $\left(\mathrm{ES}^{+}\right)$found $\left(\left[{ }^{35} \mathrm{Cl}\right] \mathrm{MH}^{+}, 368.1170\left(\mathrm{C}_{20} \mathrm{H}_{19} \mathrm{~N}_{3} \mathrm{O}_{2}{ }^{35} \mathrm{Cl}\right.\right.$ requires 368.1166$)$.

(S)-N-(1-(2-(3-chlorophenyl)hydrazineyl)-4-(methylthio)-1-oxobutan-2-yl)-1-naphthamide, 25, following the standard procedure B, tert-Butyl $N$-[(1S)-1-[N'-(3-chlorophenyl)hydrazinecarbonyl]-3-(methylsulfanyl) propyl] carbamate $(70 \mathrm{mg}, 0.23 \mathrm{mmol})$ and 1-naphthoic acid were transformed to afford the title compound as a pale yellow solid (54 mg, 56\%); m.p. 183-186 ${ }^{\circ} \mathrm{C} ; v_{\max }$ (ATR) 3239, 1667, 1635, $1594,1524,1471,1435,1313,1255,1216,1074,992,885,851,765 \mathrm{~cm}^{-1} ; \delta_{\mathrm{H}}\left(300 \mathrm{MHz}, \mathrm{DMSO}-\mathrm{d}_{6}\right)$ $10.07(1 \mathrm{H}, \mathrm{bs}, \mathrm{CONHNH}), 8.87(1 \mathrm{H}, \mathrm{d}, J$ 7, NpCONH), $8.27(1 \mathrm{H}, \mathrm{d}, J$ 8, Np-H), $8.15(1 \mathrm{H}, \mathrm{bs}$, CONHNH), 8.05-7.93 (2H, m, Np-H), $7.69(1 \mathrm{H}, \mathrm{m}, \mathrm{Np}-\mathrm{H}), 7.63-7.50(3 \mathrm{H}, \mathrm{m}, \mathrm{Np}-H), 7.17(1 \mathrm{H}$, $\mathrm{t}, J$ 6, Ar-H), $6.82(1 \mathrm{H}, \mathrm{s}, \mathrm{Ar}-\mathrm{H}), 6.76-6.70(2 \mathrm{H}, \mathrm{m}, \mathrm{Ar}-\mathrm{H}), 4.68\left(1 \mathrm{H}, \mathrm{m}, \mathrm{NHCH}\left(\mathrm{CH}_{2} \mathrm{CH}_{2} \mathrm{SCH}_{3}\right)\right)$, 2.70-2.58 (2H, m, NHCH $\left.\left(\mathrm{CH}_{2} \mathrm{CH}_{2} \mathrm{SCH}_{3}\right)\right), 2.15-2.01\left(5 \mathrm{H}, \mathrm{m}, \mathrm{NHCH}\left(\mathrm{CH}_{2} \mathrm{CH}_{2} \mathrm{SCH}_{3}\right) ; \delta_{\mathrm{C}}(75 \mathrm{MHz}\right.$, DMSO-d $_{6}$ ) 172.1 (CONHNH), 169.5 (NpCONH), 151.4 (ipso-Ar-C), 134.6 (ipso-Ar-C), 134.0 (ipso-Ar-C), 130.4 (ipso-Ar-C), $130.3(\mathrm{~Np}-\mathrm{C}), 128.6(\mathrm{~Np}-\mathrm{C}), 127.1(\mathrm{~Np}-\mathrm{C}), 126.7$ (Ar-C), 126.7 (ipso-Ar-C), 126.1 $(\mathrm{Np}-\mathrm{C}), 125.4(\mathrm{~Np}-\mathrm{C}), 118.3$ (ipso-Ar-C), $112.0(\mathrm{Ar}-\mathrm{C}), 111.4(\mathrm{Ar}-\mathrm{C}), 52.1\left(\mathrm{NHCH}\left(\mathrm{CH}_{2} \mathrm{CH}_{2} \mathrm{SCH}_{3}\right)\right.$ ), $31.4\left(\mathrm{NHCH}\left(\mathrm{CH}_{2} \mathrm{CH}_{2} \mathrm{SCH}_{3}\right)\right), 30.4\left(\mathrm{NHCH}\left(\mathrm{CH}_{2} \mathrm{CH}_{2} \mathrm{SCH}_{3}\right)\right), 15.1\left(\mathrm{NHCH}\left(\mathrm{CH}_{2} \mathrm{CH}_{2} \mathrm{SCH}_{3}\right)\right) ; \mathrm{m} / z\left(\mathrm{ES}^{+}\right)$ $428\left(\left[{ }^{35} \mathrm{Cl}\right] \mathrm{MH}^{+}\right)$, $430\left(\left[{ }^{37} \mathrm{Cl}\right] \mathrm{MH}^{+}\right)$; HRMS $\left(\mathrm{ES}^{+}\right)$found $\left[{ }^{35} \mathrm{Cl}^{2} \mathrm{MH}^{+}, 428.1192\left(\mathrm{C}_{22} \mathrm{H}_{23} \mathrm{~N}_{3} \mathrm{O}_{2} \mathrm{~S}^{35} \mathrm{Cl}\right.\right.$ requires 428.1200$)$.

(S)-N-(1-Oxo-1-(2-(4-(trifluoromethyl)phenyl)hydrazineyl)propan-2-yl)quinoline-3-carboxamide, 26, following the standard procedure B, tert-butyl (S)-(1-oxo-1-(2-(4-(trifluoromethyl) phenyl)hydrazineyl)propan-2-yl) carbamate $(0.10 \mathrm{~g}, 0.30 \mathrm{mmol})$ and 3-quinolinecarboxylic acid were transformed to afford the title compound as a white solid (53 mg, 44\%); $\mathrm{R}_{\mathrm{f}} 0.15$ (DCM/EtOH/ sat. aq. $\mathrm{NH}_{3}$ [200:8:1]); $v_{\max }$ (ATR) 3259 $(\mathrm{N}-\mathrm{H}), 2998,2935$ (C-H), 1637, 1617 (C=O), 1522, 1450, 1325, 1158, 11031065 (C-F) cm ${ }^{-1} ; \delta_{\mathrm{H}}(400 \mathrm{MHz}$, DMSO-d $\left._{6}\right) 10.09(1 \mathrm{H}, \mathrm{bs}, \mathrm{CONHNH}), 9.34(1 \mathrm{H}, \mathrm{d}, J$ 2, Quin- $H), 9.06(1 \mathrm{H}, \mathrm{d}, J$ 7, QuinCONH), $8.93(1 \mathrm{H}$, s, Quin- $H), 8.43(1 \mathrm{H}, \mathrm{s}, \mathrm{CONHNH}), 8.11(2 \mathrm{H}, \mathrm{m}$, Quin- $H), 7.89(1 \mathrm{H}, \mathrm{dd}, J$ 8, Quin- $H), 7.71(1 \mathrm{H}, \mathrm{dd}, J$, Quin- $H), 7.47\left(2 \mathrm{H}, \mathrm{d}, J\right.$ 8, Ar-H), $6.87(2 \mathrm{H}, \mathrm{d}, J$ 8, $\mathrm{Ar}-\mathrm{H}), 4.63\left(1 \mathrm{H}\right.$, quin, J 7, $\left.\mathrm{NHCH}\left(\mathrm{CH}_{3}\right) \mathrm{CO}\right), 1.50(3 \mathrm{H}, \mathrm{d}$, $J$ 7, $\left.\mathrm{NHCH}\left(\mathrm{CH}_{3}\right) \mathrm{CO}\right) ; \delta_{\mathrm{C}}\left(100 \mathrm{MHz}, \mathrm{DMSO}_{-} \mathrm{d}_{6}\right) 172.8$ (CONHNH), 165.6 (Quin-CO), 152.9 (ipso-Ar-C), 149.7 (Quin-C), 148.9 (ipso-Ar-C), 136.4 (Quin-C), 131.7 (Quin-C), 129.6 (ipso-Ar-C), 129.2 (Quin-C), 127.9 (Quin-C), 127.1 (ipso-Ar-C), 126.6 (ipso-Ar-C), 124.2 (1C, q, $\left.{ }^{1} J_{C-F} 272, \mathrm{Ar}_{-} \mathrm{CCF}_{3}\right), 118.6$ (1C, q, ${ }^{2} J_{\mathrm{C}-\mathrm{F}} 31$, ipso-Ar-CCF 3$), 111.9(\mathrm{Ar}-\mathrm{C}), 48.8\left(\mathrm{NHCH}\left(\mathrm{CH}_{3}\right) \mathrm{CO}\right), 18.1\left(\mathrm{NHCH}\left(\mathrm{CH}_{3}\right) \mathrm{CO}\right) ; \delta_{\mathrm{F}}(282 \mathrm{MHz}$, DMSO-d $\left.\mathrm{d}_{6}\right)-59.28\left(3 \mathrm{~F}, \mathrm{~s}, \mathrm{CF}_{3}\right) ; \mathrm{m} / z\left(\mathrm{ES}^{+}\right) 403\left(\mathrm{MH}^{+}\right)$; HRMS $\left(\mathrm{ES}^{+}\right)$found $\mathrm{MH}^{+}, 403.1367\left(\mathrm{C}_{20} \mathrm{H}_{18} \mathrm{~F}_{3} \mathrm{~N}_{4} \mathrm{O}_{2}\right.$ requires 403.1382).

(S)-N-(4-(methylthio)-1-oxo-1-(2-(4-(trifluoromethyl)phenyl)hydrazineyl)butan-2-yl)quinoline-3-carboxamide, 27, following the standard procedure B, tert-Butyl $N-[(1 S)-3$-(methylsulfanyl)-1-\{N'-[4-(trifluoromethyl) phenyl]hydrazine carbonyl propyl]carbamate $(65 \mathrm{mg}, 0.19 \mathrm{mmol})$ and 3-quinolinecarboxylic acid were transformed to afford the title compound as a beige solid $(57 \mathrm{mg}, 65 \%) ; \mathrm{m} . \mathrm{p} .138-141{ }^{\circ} \mathrm{C} ; v_{\max }$ (ATR) 3258, 3001, 1636, 1616, 1538, 1496, 1418, 1328, 1231, 1185, 1158, 1098, 1066, 1009, 959, 917, 902, 865, $832 \mathrm{~cm}^{-1} ; \delta_{\mathrm{H}}\left(300 \mathrm{MHz}, \mathrm{DMSO}-\mathrm{d}_{6}\right) 10.15(1 \mathrm{H}, \mathrm{bs}, \mathrm{CONHNH}), 9.34(1 \mathrm{H}, \mathrm{d}, J 2$, Quin-H), 9.06 
(1H, d, J 7, QuinCONH), $8.93(1 \mathrm{H}, \mathrm{s}$, Quin- $H), 8.45(1 \mathrm{H}, \mathrm{s}, \mathrm{CONHNH}), 8.12(2 \mathrm{H}, \mathrm{m}$, Quin- $H), 7.88$ $(1 \mathrm{H}, \mathrm{dd}, J$ 8, Quin- $H), 7.71(1 \mathrm{H}, \mathrm{dd}, J$ 8, Quin- $H), 7.47(2 \mathrm{H}, \mathrm{d}, J$ 8, Ar-H), $6.85(2 \mathrm{H}, \mathrm{d}, J$ 8, Ar-H), $4.70\left(1 \mathrm{H}, \mathrm{m}, \mathrm{NHCH}\left(\mathrm{CH}_{2} \mathrm{CH}_{2} \mathrm{SCH}_{3}\right)\right), 2.75-2.56\left(2 \mathrm{H}, \mathrm{m}, \mathrm{NHCH}\left(\mathrm{CH}_{2} \mathrm{CH}_{2} \mathrm{SCH}_{3}\right)\right), 2.20-2.05(5 \mathrm{H}, \mathrm{m}$, $\mathrm{NHCH}\left(\mathrm{CH}_{2} \mathrm{CH}_{2} \mathrm{SCH}_{3}\right) ; \delta_{\mathrm{C}}\left(75 \mathrm{MHz}, \mathrm{DMSO}-\mathrm{d}_{6}\right) 171.9$ (CONHNH), 166.0 (Quin-CO), 152.9 (ipso-Ar-C), 149.7 (Quin-C), 148.9 (ipso-Ar-C), 136.4 (Quin-C), 131.7 (Quin-C), 129.6 (ipso-Ar-C), 129.3 (Quin-C), 127.9 (Quin-C), 127.1 (ipso-Ar-C), 126.9 (Ar-C), 123.7 (1C, q, $\left.{ }^{1} J_{\text {C-F }} 270, \mathrm{Ar}_{-} \mathrm{CCF}_{3}\right), 118.6\left(1 \mathrm{C}, \mathrm{q},{ }^{2} J_{\mathrm{C}-\mathrm{F}}\right.$ 31, ipso- $\left.\mathrm{Ar}-\mathrm{CCF}_{3}\right), 112.0(\mathrm{Ar}-\mathrm{C}), 52.4\left(\mathrm{NHCH}\left(\mathrm{CH}_{2} \mathrm{CH}_{2} \mathrm{SCH}_{3}\right)\right), 31.4\left(\mathrm{NHCH}\left(\mathrm{CH}_{2} \mathrm{CH}_{2} \mathrm{SCH}_{3}\right)\right), 30.5$ $\left(\mathrm{NHCH}\left(\mathrm{CH}_{2} \mathrm{CH}_{2} \mathrm{SCH}_{3}\right)\right), 15.1\left(\mathrm{NHCH}\left(\mathrm{CH}_{2} \mathrm{CH}_{2} \mathrm{SCH}_{3}\right)\right) ; \delta_{\mathrm{F}}\left(282 \mathrm{MHz}, \mathrm{DMSO}-\mathrm{d}_{6}\right)-59.27\left(3 \mathrm{~F}, \mathrm{~s}, \mathrm{CF}_{3}\right)$; $m / z\left(\mathrm{ES}^{+}\right) 463\left(\mathrm{MH}^{+}\right) ; \mathrm{HRMS}\left(\mathrm{ES}^{+}\right)$found $\mathrm{MH}^{+}, 463.1416\left(\mathrm{C}_{22} \mathrm{H}_{22} \mathrm{~F}_{3} \mathrm{~N}_{4} \mathrm{O}_{2} \mathrm{~S}\right.$ requires 463.1416).

(S)-N-(1-(2-(3-chlorophenyl)hydrazineyl)-1-oxopropan-2-yl)quinoline-3-carboxamide, 28 , following the standard procedure B, tert-butyl (S)-(1-(2-(3-chlorophenyl)hydrazineyl)-1-oxopropan-2-yl)carbamate $(0.10 \mathrm{~g}, 0.32 \mathrm{mmol})$ and 3-quinolinecarboxylic acid were transformed to afford the title compound as a white solid (37 mg, 30\%); $\mathrm{R}_{\mathrm{f}} 0.29\left(\mathrm{DCM} / \mathrm{EtOH} / \mathrm{sat}\right.$. aq. $\mathrm{NH}_{3}$ [200:8:1]); $v_{\max }$ (ATR) $3324(\mathrm{~N}-\mathrm{H}), 2988$, $2947(\mathrm{C}-\mathrm{H}), 1646,1613(\mathrm{C}=\mathrm{O}), 1513(\mathrm{C}=\mathrm{N}), 1151,1103,1065(\mathrm{C}-\mathrm{F}) \mathrm{cm}^{-1} ; \delta_{\mathrm{H}}\left(400 \mathrm{MHz}, \mathrm{DMSO}-\mathrm{d}_{6}\right) 10.00$ $(1 \mathrm{H}, \mathrm{bs}, \mathrm{CONHNH}), 9.35(1 \mathrm{H}, \mathrm{d}, J$ 2, Quin-NCH), 9.08 (1H, d, J 7, Quin-CONH), 8.93 (1H, s, Quin-CH), 8.15-8.07 (3H, m, Quin-CH, CONHNH), 7.89 (1H, t, J 8, Quin-CH), 7.72 (1H, t, J 8, Quin-CH), 7.15 $(1 \mathrm{H}, \mathrm{t}, J$ 8, $\mathrm{Ar}-\mathrm{H}), 6.78(1 \mathrm{H}, \mathrm{t}, J$ 2, $\mathrm{Ar}-\mathrm{H}), 6.71(2 \mathrm{H}, \mathrm{m}, \mathrm{Ar}-\mathrm{H}), 4.60\left(1 \mathrm{H}, \mathrm{m}, \mathrm{NHCH}\left(\mathrm{CH}_{3}\right)\right), 1.48(3 \mathrm{H}, \mathrm{d}, J$ 7, $\left.\mathrm{NHCH}\left(\mathrm{CH}_{3}\right)\right) ; \delta_{\mathrm{C}}\left(75 \mathrm{MHz}, \mathrm{DMSO}-\mathrm{d}_{6}\right) 172.7$ (CONHNH), 165.6 (Quin-CONH), 151.4 (ipso-Ar-C), 149.7 (Quin-C), 149.0 (ipso-Quin-C), 136.4 (Quin-C), 134.0 (ipso-Ar-C), 131.7 (Quin-C), 130.8 (Ar-C), 129.6 (Quin-C), 129.3 (Quin-C), 127.9 (Quin-C), 127.1 (ipso-Ar-C), 126.9 (ipso-Ar-C), 118.3 (Ar-C), 111.9 (Ar-C), $111.3(\mathrm{Ar}-\mathrm{C}), 48.7\left(\mathrm{NHCH}\left(\mathrm{CH}_{3}\right)\right), 18.1\left(\mathrm{NHCH}\left(\mathrm{CH}_{3}\right)\right) ; \mathrm{m} / z\left(\mathrm{ES}^{+}\right) 369\left({ }^{35} \mathrm{Cl}^{-} \mathrm{MH}^{+}\right), 371$ $\left(\left[{ }^{37} \mathrm{Cl}\right] \mathrm{MH}^{+}\right)$; HRMS $\left(\mathrm{ES}^{+}\right)$found $\left[{ }^{35} \mathrm{Cl}\right] \mathrm{MH}^{+}, 369.1120\left(\mathrm{C}_{19} \mathrm{H}_{18} \mathrm{~N}_{4} \mathrm{O}_{2}{ }^{35} \mathrm{Cl}\right.$ requires 369.1118).

(S)-N-(1-(2-(3-chlorophenyl)hydrazineyl)-4-(methylthio)-1-oxobutan-2-yl)quinoline-3-carboxamide, 29, following the standard procedure B, tert-Butyl $N-\left[(1 S)-1-\left[N^{\prime}\right.\right.$-(3-chlorophenyl)hydrazinecarbonyl]-3(methylsulfanyl)propyl] carbamate $(70 \mathrm{mg}, 0.23 \mathrm{mmol}$ ) and 3-quinolinecarboxylic acid were transformed to afford the title compound as a white solid (68 mg, 71\%); m.p. 141-144 ${ }^{\circ} \mathrm{C}$; $v_{\max }$ (ATR) 3211, 3029, 2913, 1664, 1638, 1595, 1570, 1534, 1492, 1424, 1374, 1307, 1272, 1229, 1203, 1155, 1136, 1112, 1080, $1019,993,981,960,948,914,900,876,855 \mathrm{~cm}^{-1} ; \delta_{\mathrm{H}}\left(400 \mathrm{MHz}, \mathrm{DMSO}-\mathrm{d}_{6}\right) 10.08(1 \mathrm{H}, \mathrm{bs}, \mathrm{CONHNH})$, $9.35(1 \mathrm{H}, \mathrm{d}, J$ 2, Quin-NCH), $9.07(1 \mathrm{H}, \mathrm{d}, J$ 7, Quin-CONH), $8.93(1 \mathrm{H}, \mathrm{s}$, Quin- $\mathrm{CH}), 8.15-8.07(3 \mathrm{H}, \mathrm{m}$, Quin-CH, CONHNH), $7.89(1 \mathrm{H}, \mathrm{t}, J$ 8, Quin-CH), $7.72(1 \mathrm{H}, \mathrm{t}, J$ 8, Quin-CH), $7.15(1 \mathrm{H}, \mathrm{t}, J$ 8, Ar-H), $6.77(1 \mathrm{H}, \mathrm{t}, J 2, \mathrm{Ar}-\mathrm{H}), 6.70(2 \mathrm{H}, \mathrm{m}, \mathrm{Ar}-\mathrm{H}), 4.67\left(1 \mathrm{H}, \mathrm{m}, \mathrm{NHCH}\left(\mathrm{CH}_{2} \mathrm{CH}_{2} \mathrm{SCH}_{3}\right)\right), 2.71-2.59(2 \mathrm{H}, \mathrm{m}$, $\left.\mathrm{NHCH}\left(\mathrm{CH}_{2} \mathrm{CH}_{2} \mathrm{SCH}_{3}\right)\right)$, 2.16-2.06 $\left(5 \mathrm{H}, \mathrm{m}, \mathrm{NHCH}\left(\mathrm{CH}_{2} \mathrm{CH}_{2} \mathrm{SCH}_{3}\right) ; \delta_{\mathrm{C}}\left(75 \mathrm{MHz}, \mathrm{DMSO}-\mathrm{d}_{6}\right) 171.8\right.$ (CONHNH), 166.0 (Quin-CONH), 151.4 (ipso-Ar-C), 149.7 (Quin-C), 149.0 (ipso-Quin-C), 136.4 (Quin-C), 134.0 (ipso-Ar-C), 131.7 (Quin-C), 130.8 (Ar-C), 129.6 (Quin-C), 129.3 (Quin-C), 127.9 (Quin-C), 127.1 (ipso-Ar-C), 126.9 (ipso-Ar-C), $118.3(\mathrm{Ar}-\mathrm{C}), 111.9(\mathrm{Ar}-\mathrm{C}), 111.4(\mathrm{Ar}-\mathrm{C}), 52.4\left(\mathrm{NHCH}\left(\mathrm{CH}_{2} \mathrm{CH}_{2} \mathrm{SCH}_{3}\right)\right.$ ), $31.4\left(\mathrm{NHCH}\left(\mathrm{CH}_{2} \mathrm{CH}_{2} \mathrm{SCH}_{3}\right)\right), 30.4\left(\mathrm{NHCH}\left(\mathrm{CH}_{2} \mathrm{CH}_{2} \mathrm{SCH}_{3}\right)\right), 15.1\left(\mathrm{NHCH}\left(\mathrm{CH}_{2} \mathrm{CH}_{2} \mathrm{SCH}_{3}\right)\right) ; \mathrm{m} / z\left(\mathrm{ES}^{+}\right)$ $429\left(\left[{ }^{35} \mathrm{Cl}\right] \mathrm{MH}^{+}\right)$, $431\left(\left[{ }^{37} \mathrm{Cl}\right] \mathrm{MH}^{+}\right)$; HRMS $\left(\mathrm{ES}^{+}\right)$found $\left[{ }^{35} \mathrm{Cl} \mathrm{MH}^{+}, 429.1148\left(\mathrm{C}_{21} \mathrm{H}_{22}{ }^{35} \mathrm{ClN}_{4} \mathrm{O}_{2} \mathrm{~S}\right.\right.$ requires 429.1152$)$.

(S)-3,5-Dinitro-N-(1-oxo-1-(2-(4-(trifluoromethyl)phenyl)hydrazineyl)propan-2-yl)benzamide, 30, following the standard procedure B, tert-butyl (S)-(1-oxo-1-(2-(4-(trifluoromethyl) phenyl)hydrazineyl)propan -2-yl)carbamate $(0.10 \mathrm{~g}, 0.30 \mathrm{mmol})$ and 3,5-dinitrobenzoic acid were transformed to afford the title compound as a white solid (79 mg, 60\%); $v_{\max }(\mathrm{ATR}) 3250(\mathrm{NH}), 3101(\mathrm{Ar}-\mathrm{CH}), 2896(\mathrm{CH}), 1641(\mathrm{C}=\mathrm{O})$, 1617 (Ar C-C), 1535, 1342, 1330, 1160, 1107, 1066 (C-F) cm ${ }^{-1}$; $\delta_{\mathrm{H}}\left(400 \mathrm{MHz}, \mathrm{DMSO}-\mathrm{d}_{6}\right) 10.13(1 \mathrm{H}, \mathrm{bs}$, CONHNH), $9.47(1 \mathrm{H}, \mathrm{d}, J$ 7, ArCONH), $9.14(2 \mathrm{H}, \mathrm{d}, J$ 2, Ar-H), $8.98(1 \mathrm{H}, \mathrm{dd}, J$ 2, Ar-H), $8.43(1 \mathrm{H}$, bs, CONHNH), $7.47\left(2 \mathrm{H}, \mathrm{d}, J\right.$ 8, Ar-H), $6.84(2 \mathrm{H}, \mathrm{d}, J$ 8, $\mathrm{Ar}-\mathrm{H}), 4.61\left(1 \mathrm{H}, \mathrm{m}, \mathrm{NHCH}\left(\mathrm{CH}_{3}\right) \mathrm{CO}\right), 1.49$ $\left(3 \mathrm{H}, \mathrm{d}, J \mathrm{~J}, \mathrm{NHCH}\left(\mathrm{CH}_{3}\right) \mathrm{CO}\right) ; \delta_{\mathrm{C}}\left(100 \mathrm{MHz}, \mathrm{DMSO}_{-} \mathrm{d}_{6}\right) 172.4(\mathrm{CONHNH}), 163.0(\mathrm{ArCONH}), 152.9$ (ipso-Ar-C), 148.6 (ipso-Ar-C), 137.1 (ipso-Ar-C), 128.4 (Ar-C), 126.7 (Ar-C), 121.5 (Ar-C), 111.9 (Ar-C), 
$49.2\left(\mathrm{NHCH}\left(\mathrm{CH}_{3}\right) \mathrm{CO}\right), 18.0\left(\mathrm{NHCH}\left(\mathrm{CH}_{3}\right) \mathrm{CO}\right) ; \delta_{\mathrm{F}}\left(282 \mathrm{MHz}, \mathrm{DMSO}-\mathrm{d}_{6}\right)-59.34\left(3 \mathrm{~F}, \mathrm{~s}, \mathrm{CF}_{3}\right) ; m / z\left(\mathrm{ES}^{+}\right)$ $442\left(\mathrm{MH}^{+}\right)$; HRMS $\left(\mathrm{ES}^{+}\right)$found $\mathrm{MH}^{+}, 442.0958\left(\mathrm{C}_{17} \mathrm{H}_{15} \mathrm{~F}_{3} \mathrm{~N}_{5} \mathrm{O}_{6}\right.$ requires 442.0974).

(S)-N-(4-(methylthio)-1-oxo-1-(2-(4-(trifluoromethyl)phenyl)hydrazineyl)butan-2-yl)-3,5-dinitrobenzamide, 31, following the standard procedure B, tert-Butyl $N-[(1 S)-3$-(methylsulfanyl)-1-\{N'-[4-(trifluoromethyl) phenyl]hydrazine carbonyl $\}$ propyl]carbamate $(65 \mathrm{mg}, 0.19 \mathrm{mmol})$ and 3,5-dinitrobenzoic acid were transformed to afford the title compound as a beige solid (36 mg, 38\%); m.p. 97-100 ${ }^{\circ} \mathrm{C} ; v_{\max }$ (ATR) $3464,3212,3125,3075,1662,1633,1614,1541,1440,1342,1322,1251,1162,1115,1063,1010,933$, $837 \mathrm{~cm}^{-1} ; \delta_{\mathrm{H}}\left(300 \mathrm{MHz}, \mathrm{DMSO}-\mathrm{d}_{6}\right) 10.20(1 \mathrm{H}, \mathrm{bs}, \mathrm{CONHNH}), 9.45(1 \mathrm{H}, \mathrm{d}, J$ 7, ArCONH), 9.15 $(2 \mathrm{H}, \mathrm{d}, J$ 2, $\mathrm{Ar}-\mathrm{H}), 8.99(1 \mathrm{H}, \mathrm{dd}, J$ 2, $\mathrm{Ar}-\mathrm{H}), 8.44(1 \mathrm{H}, \mathrm{bs}, \mathrm{CONHNH}), 7.47(2 \mathrm{H}, \mathrm{d}, J$ 8, Ar-H), 6.84 $(2 \mathrm{H}, \mathrm{d}, \mathrm{J} 8, \mathrm{Ar}-\mathrm{H}), 4.68\left(1 \mathrm{H}, \mathrm{m}, \mathrm{NHCH}\left(\mathrm{CH}_{2} \mathrm{CH}_{2} \mathrm{SCH}_{3}\right)\right), 2.74-2.53\left(2 \mathrm{H}, \mathrm{m}, \mathrm{NHCH}\left(\mathrm{CH}_{2} \mathrm{CH}_{2} \mathrm{SCH}_{3}\right)\right)$, 2.19-2.04 (5H, m, $\mathrm{NHCH}\left(\mathrm{CH}_{2} \mathrm{CH}_{2} \mathrm{SCH}_{3}\right) ; \delta_{\mathrm{C}}\left(75 \mathrm{MHz}, \mathrm{DMSO}-\mathrm{d}_{6}\right) 171.4(\mathrm{CONHNH}), 163.4(\mathrm{ArCONH})$, 152.8 (ipso-Ar-C), 148.6 (ipso-Ar-C), 137.0 (ipso-Ar-C), 128.5 (Ar-C), 127.3 (ipso-Ar-C), 126.6 (Ar-C), 121.5 (Ar-C), 118.7 (1C, q ${ }^{2} J_{\mathrm{C}-\mathrm{F}} 32$, ipso-Ar- $\left.\mathrm{CCF}_{3}\right), 111.9(\mathrm{Ar}-\mathrm{C}), 52.7\left(\mathrm{NHCH}\left(\mathrm{CH}_{2} \mathrm{CH}_{2} \mathrm{SCH}_{3}\right)\right), 31.2$ $\left(\mathrm{NHCH}\left(\mathrm{CH}_{2} \mathrm{CH}_{2} \mathrm{SCH}_{3}\right)\right), 30.4\left(\mathrm{NHCH}\left(\mathrm{CH}_{2} \mathrm{CH}_{2} \mathrm{SCH}_{3}\right)\right), 15.1\left(\mathrm{NHCH}\left(\mathrm{CH}_{2} \mathrm{CH}_{2} \mathrm{SCH}_{3}\right)\right) ; \delta_{\mathrm{F}}(282 \mathrm{MHz}$, DMSO-d $\left.\mathrm{d}_{6}\right)-59.30\left(3 \mathrm{~F}, \mathrm{~s}, \mathrm{CF}_{3}\right) ; \mathrm{m} / z\left(\mathrm{ES}^{+}\right) 502\left(\mathrm{MH}^{+}\right)$; HRMS $\left(\mathrm{ES}^{+}\right)$found $\mathrm{MH}^{+}, 502.1010\left(\mathrm{C}_{19} \mathrm{H}_{19} \mathrm{~F}_{3} \mathrm{~N}_{5} \mathrm{O}_{6} \mathrm{~S}\right.$ requires 502.1008).

(S)-N-(1-(2-(3-chlorophenyl)hydrazineyl)-1-oxopropan-2-yl)-3,5-dinitrobenzamide, 32, following the standard procedure B, tert-butyl (S)-(1-(2-(3-chlorophenyl)hydrazineyl)-1-oxopropan-2-yl)carbamate $(0.10 \mathrm{~g}, 0.32 \mathrm{mmol})$ and 3,5-dinitrobenzoic acid were transformed to afford the title compound as a light brown solid (54 mg, 42\%); $\mathrm{R}_{\mathrm{f}} 0.21$ (DCM/EtOH/ sat. aq. $\mathrm{NH}_{3}$ [200:8:1]); $v_{\max }(\mathrm{ATR}) 3264(\mathrm{NH})$, $3094(\mathrm{Ar}-\mathrm{CH}), 2983,2926(\mathrm{CH}), 1651,1597(\mathrm{C}=\mathrm{O}), 1537,1342(\mathrm{~N}-\mathrm{O}), 1334(\mathrm{C}=\mathrm{C}) \mathrm{cm}^{-1}$; $\delta_{\mathrm{H}}(300 \mathrm{MHz}$, DMSO-d $\left._{6}\right) 10.05(1 \mathrm{H}, \mathrm{bs}, \mathrm{CONHNH}), 9.48(1 \mathrm{H}, \mathrm{d}, J$ 7, ArCONH), $9.15(2 \mathrm{H}, \mathrm{d}, \mathrm{J} 2, \mathrm{Ar}-\mathrm{H}), 8.99(1 \mathrm{H}$, $\mathrm{m}, \mathrm{Ar}-\mathrm{H}), 8.10(1 \mathrm{H}, \mathrm{bs}, \mathrm{CONHNH}), 7.14(1 \mathrm{H}, \mathrm{t}, J$ 8, Ar-H), $6.76(1 \mathrm{H}, \mathrm{t}, J$ 2, Ar-H), 6.73-6.66 (2H, m, $\mathrm{Ar}-\mathrm{H}), 4.57\left(1 \mathrm{H}, \mathrm{m}, \mathrm{NHCH}\left(\mathrm{CH}_{3}\right) \mathrm{CO}\right), 1.48\left(3 \mathrm{H}, \mathrm{d}, J\right.$ 7, $\left.\mathrm{NHCH}\left(\mathrm{CH}_{3}\right) \mathrm{CO}\right) ; \delta_{\mathrm{C}}\left(100 \mathrm{MHz}, \mathrm{DMSO}-\mathrm{d}_{6}\right)$ 172.3 (CONHNH), 163.0 (ArCONH), 151.3 (ipso-Ar-C), 148.5 (ipso-Ar-C), 137.1 (ipso-Ar-C), 134.0 (ipso-Ar-C), 130.7 (Ar-C), 128.4 (Ar-C), 118.3 (Ar-C), 111.9 (Ar-C), $111.3(\mathrm{Ar}-\mathrm{C}), 49.2\left(\mathrm{NHCH}\left(\mathrm{CH}_{3}\right) \mathrm{CO}\right)$, $17.9\left(\mathrm{NHCH}\left(\mathrm{CH}_{3}\right) \mathrm{CO}\right) ; \mathrm{m} / z\left(\mathrm{ES}^{+}\right) 408\left(\left[{ }^{35} \mathrm{Cl}\right] \mathrm{MH}^{+}\right), 410\left(\left[{ }^{37} \mathrm{Cl}\right] \mathrm{MH}^{+}\right)$; $\left.\mathrm{HRMS}_{(\mathrm{ES}}{ }^{+}\right)$found $\left[{ }^{35} \mathrm{Cl}\right] \mathrm{MH}^{+}$, $408.0701\left(\mathrm{C}_{16} \mathrm{H}_{15} \mathrm{~N}_{5} \mathrm{O}_{6}{ }^{35} \mathrm{Cl}\right.$ requires 408.0711).

(S)-N-(1-(2-(3-chlorophenyl)hydrazineyl)-4-(methylthio)-1-oxobutan-2-yl)-3,5-dinitrobenzamide, 33, following the standard procedure B, tert-Butyl $N$-[(1S)-1-[N'-(3-chlorophenyl)hydrazinecarbonyl]-3(methylsulfanyl)propyl] carbamate $(100 \mathrm{mg}, 0.32 \mathrm{mmol})$ and 3,5-dinitrobenzoic acid were transformed to afford the title compound as a light brown gum (149 mg, 99\%); m.p. $87-89{ }^{\circ} \mathrm{C} ; v_{\max }$ (ATR) $3259,3071,1649,1640,1596,1536,1476,1341,1340,1320,1076,1041,918,847 \mathrm{~cm}^{-1} ; \delta_{\mathrm{H}}(300 \mathrm{MHz}$, DMSO-d $\left._{6}\right) 10.12(1 \mathrm{H}, \mathrm{bs}, \mathrm{CONHNH}), 9.44(1 \mathrm{H}, \mathrm{d}, J$ 7, ArCONH), $9.15(2 \mathrm{H}, \mathrm{d}, J$ 2, Ar-H), 8.99 $(1 \mathrm{H}, \mathrm{dd}, J$ 2, Ar-H), $8.10(1 \mathrm{H}, \mathrm{bs}, \mathrm{CONHNH}), 7.15(1 \mathrm{H}, \mathrm{t}, J$ 8, Ar-H), 6.78-6.62 $(3 \mathrm{H}, \mathrm{m}, \mathrm{Ar}-\mathrm{H})$, $4.68\left(1 \mathrm{H}, \mathrm{m}, \mathrm{NHCH}\left(\mathrm{CH}_{2} \mathrm{CH}_{2} \mathrm{SCH}_{3}\right)\right), 2.74-2.53\left(2 \mathrm{H}, \mathrm{m}, \mathrm{NHCH}\left(\mathrm{CH}_{2} \mathrm{CH}_{2} \mathrm{SCH}_{3}\right)\right), 2.19-2.04(5 \mathrm{H}, \mathrm{m}$, $\mathrm{NHCH}\left(\mathrm{CH}_{2} \mathrm{CH}_{2} \mathrm{SCH}_{3}\right) ; \delta_{\mathrm{C}}\left(75 \mathrm{MHz}, \mathrm{DMSO}_{6}\right) 171.4(\mathrm{CONHNH}), 163.4$ (ArCONH), 151.2 (ipso-Ar-C), 148.6 (ipso-Ar-C), 137.1 (ipso-Ar-C), 134.0 (ipso-Ar-C), 130.7 (Ar-C), 128.5 (Ar-C), 121.3 (Ar-C), 118.5 (Ar-C), $111.9(\mathrm{Ar}-\mathrm{C}), 111.3(\mathrm{Ar}-\mathrm{C}), 52.8\left(\mathrm{NHCH}\left(\mathrm{CH}_{2} \mathrm{CH}_{2} \mathrm{SCH}_{3}\right)\right), 31.2\left(\mathrm{NHCH}\left(\mathrm{CH}_{2} \mathrm{CH}_{2} \mathrm{SCH}_{3}\right)\right), 30.4$ $\left(\mathrm{NHCH}\left(\mathrm{CH}_{2} \mathrm{CH}_{2} \mathrm{SCH}_{3}\right)\right), 15.1\left(\mathrm{NHCH}\left(\mathrm{CH}_{2} \mathrm{CH}_{2} \mathrm{SCH}_{3}\right)\right) ; \mathrm{m} / \mathrm{z}\left(\mathrm{ES}^{+}\right) 468\left(\left[{ }^{35} \mathrm{Cl}\right] \mathrm{MH}^{+}\right), 470\left(\left[{ }^{37} \mathrm{Cl}\right] \mathrm{MH}^{+}\right)$; HRMS $\left(\mathrm{ES}^{+}\right)$found $\left[{ }^{35} \mathrm{Cl}\right] \mathrm{MH}^{+}, 468.0743\left(\mathrm{C}_{18} \mathrm{H}_{19}{ }^{35} \mathrm{ClN}_{5} \mathrm{O}_{6} \mathrm{~S}\right.$ requires 468.0745).

(S)-6-chloro-2-ethyl-N-(1-oxo-1-(2-(4-(trifluoromethyl)phenyl)hydrazineyl)propan-2-yl)imidazo[1,2-a] pyridine-3-carboxamide, 34, following the standard procedure B, tert-butyl(S)-(1-oxo-1-(2-(4-(trifluoromethyl) phenyl)hydrazineyl)propan-2-yl)carbamate $(0.1 \mathrm{~g}, 0.35 \mathrm{mmol})$ and 6-chloro-2-ethylimidazo[1,2-a]pyridine -3-carboxylic acid were transformed to afford the title compound as a pale yellow solid (69 $\mathrm{mg}, 43 \%$ ); m.p. $221-224^{\circ} \mathrm{C}$; $v_{\max }$ (ATR) 3252, 2967, 2932, 1667, 1609, 1541, 1522, 1493, 1453, 1442, 1391, 1329, $1281,1251,1220,1160,1097,1066,1009,995,952,906 \mathrm{~cm}^{-1} ; \delta_{\mathrm{H}}\left(400 \mathrm{MHz}, \mathrm{DMSO}-\mathrm{d}_{6}\right) 10.10(1 \mathrm{H}, \mathrm{d}, J 2$, 
CONHNH), $9.01(1 \mathrm{H}, \mathrm{d}, J$ 2, Ar-H), 8.47 (1H, d, J 2, CONHNH), $8.33(1 \mathrm{H}, \mathrm{d}, J$ 7, NHCH(CH 3$) \mathrm{CO}), 7.68$ $(1 \mathrm{H}, \mathrm{d}, J$ 9, $\mathrm{Ar}-\mathrm{H}), 7.51-7.45(3 \mathrm{H}, \mathrm{m}, \mathrm{Ar}-\mathrm{H}), 6.91(2 \mathrm{H}, \mathrm{d}, J$ 8, $\mathrm{Ar}-\mathrm{H}), 4.60\left(1 \mathrm{H}, \mathrm{q}, J\right.$ J, $\left.\mathrm{NHCH}\left(\mathrm{CH}_{3}\right) \mathrm{CO}\right)$, $3.00\left(2 \mathrm{H}, \mathrm{q}, J 7, \mathrm{CH}_{2} \mathrm{CH}_{3}\right), 1.46\left(3 \mathrm{H}, \mathrm{d}, J\right.$ 7, $\left.\mathrm{NHCH}\left(\mathrm{CH}_{3}\right) \mathrm{CO}\right), 1.26\left(3 \mathrm{H}, \mathrm{t}, J 7, \mathrm{CH}_{2} \mathrm{CH}_{3}\right) ; \delta_{\mathrm{C}}(100 \mathrm{MHz}$, DMSO-d $\left.{ }_{6}\right) 172.8$ (CONHNH), $161.1(\mathrm{CONH}), 153.0$ (ipso-Ar-C), 151.8 (ipso-Ar-C), 143.7 (ipso-Ar-C), 127.5 (ipso-Ar-C), 126.6 (Ar-C), 125.2 (Ar-C), 120.1 (ipso-Ar-C), 117.7 (Ar-C), 112.0 (Ar-C), 48.3 $\left(\mathrm{NHCH}\left(\mathrm{CH}_{3}\right) \mathrm{CO}\right), 22.2\left(\mathrm{CH}_{2} \mathrm{CH}_{3}\right), 18.2\left(\mathrm{NHCH}\left(\mathrm{CH}_{3}\right) \mathrm{CO}\right), 13.5\left(\mathrm{CH}_{2} \mathrm{CH}_{3}\right) ; m / z\left(\mathrm{ES}^{+}\right) 454\left(\left[{ }^{35} \mathrm{Cl}_{\mathrm{MH}} \mathrm{MH}^{+}\right)\right.$, $456\left(\left[{ }^{37} \mathrm{Cl}\right] \mathrm{MH}^{+}\right)$; HRMS $\left(\mathrm{ES}^{+}\right)$found $\left[{ }^{35} \mathrm{Cl}\right] \mathrm{MH}^{+}, 454.1256\left(\mathrm{C}_{20} \mathrm{H}_{20}{ }^{35} \mathrm{ClF}_{3} \mathrm{~N}_{5} \mathrm{O}_{2}\right.$ requires 454.1258).

(S)-6-chloro-2-ethyl-N-(4-(methylthio)-1-oxo-1-(2-(4-(trifluoromethyl)phenyl)hydrazineyl)butan-2-yl) imidazo[1,2-a]pyridine-3-carboxamide, 35, following the standard procedure B, tert-Butyl $N$-[(1S)-3-(methylsulfanyl)-1-\{N'-[4-(trifluoromethyl)phenyl]hydrazine carbonyl\}propyl]carbamate (65 mg, $0.19 \mathrm{mmol}$ ) and 6-chloro-2-ethylimidazo[1,2-a]pyridine-3-carboxylic acid were transformed to afford the title compound as a white solid (53 mg, 55\%); m.p. 227-230 ${ }^{\circ} \mathrm{C}$; $v_{\max }$ (ATR) 3248,1664 , $1612,1526,1487,1417,1394,1374,1328,1291,1189,1158,1108,1066,1013,1000,924,897,838 \mathrm{~cm}^{-1} ; \delta_{\mathrm{H}}$ (300 MHz, DMSO-d 6$) 10.16(1 \mathrm{H}, \mathrm{d}, J$ 2, CONHNH), $8.96(1 \mathrm{H}, \mathrm{d}, J 2$ 2, Ar-H), $8.48(1 \mathrm{H}, \mathrm{d}, J$ 2, CONHNH), $8.42(1 \mathrm{H}, \mathrm{d}, J$ 7, ArCONH), $7.67(1 \mathrm{H}, \mathrm{d}, J$ 9 , Ar-H), 7.52-7.45 (3H, m, Ar-H), $6.92(2 \mathrm{H}, \mathrm{d}, J$ 8, Ar-H), 4.70 $\left(1 \mathrm{H}, \mathrm{m}, \mathrm{NHCH}\left(\mathrm{CH}_{2} \mathrm{CH}_{2} \mathrm{SCH}_{3}\right)\right), 3.00\left(2 \mathrm{H}, \mathrm{q}, J 7, \mathrm{CH}_{2} \mathrm{CH}_{3}\right), 2.75-2.56\left(2 \mathrm{H}, \mathrm{m}, \mathrm{NHCH}\left(\mathrm{CH}_{2} \mathrm{CH}_{2} \mathrm{SCH}_{3}\right)\right)$, 2.20-2.05 (5H, m, NHCH $\left(\mathrm{CH}_{2} \mathrm{CH}_{2} \mathrm{SCH}_{3}\right), 1.28\left(3 \mathrm{H}, \mathrm{t}, J\right.$ 7, $\left.\mathrm{CH}_{2} \mathrm{CH}_{3}\right) ; \delta_{\mathrm{C}}\left(75 \mathrm{MHz}, \mathrm{DMSO}-\mathrm{d}_{6}\right) 171.9$ (CONHNH), $161.6(\mathrm{CONH}), 152.9$ (ipso-Ar-C), 151.8 (ipso-Ar-C), 143.7 (ipso-Ar-C), 127.5 (ipso-Ar-C), 126.5 (Ar-C), 125.1 (Ar-C), 120.1 (ipso-Ar-C), 117.7 (Ar-C), 116.3 (ipso-Ar-C), 112.0 (Ar-C), 52.4 $\left(\mathrm{NHCH}\left(\mathrm{CH}_{2} \mathrm{CH}_{2} \mathrm{SCH}_{3}\right)\right), 31.2\left(\mathrm{NHCH}\left(\mathrm{CH}_{2} \mathrm{CH}_{2} \mathrm{SCH}_{3}\right)\right), 30.4\left(\mathrm{NHCH}\left(\mathrm{CH}_{2} \mathrm{CH}_{2} \mathrm{SCH}_{3}\right)\right), 22.2\left(\mathrm{CH}_{2} \mathrm{CH}_{3}\right)$, $15.1\left(\mathrm{NHCH}\left(\mathrm{CH}_{2} \mathrm{CH}_{2} \mathrm{SCH}_{3}\right)\right), 13.4\left(\mathrm{CH}_{2} \mathrm{CH}_{3}\right) ; \delta_{\mathrm{F}}\left(282 \mathrm{MHz}, \mathrm{DMSO}-\mathrm{d}_{6}\right)-59.32\left(3 \mathrm{~F}, \mathrm{~s}, \mathrm{CF}_{3}\right) ; \mathrm{m} / z\left(\mathrm{ES}^{+}\right)$ $\left.514\left(\left[{ }^{35} \mathrm{Cl}\right] \mathrm{MH}^{+}\right), 516\left({ }^{37} \mathrm{Cl}\right] \mathrm{MH}^{+}\right)$; HRMS $\left(\mathrm{ES}^{+}\right)$found $\left.{ }^{35} \mathrm{Cl}\right] \mathrm{MH}^{+}, 514.1290\left(\mathrm{C}_{22} \mathrm{H}_{24}{ }^{35} \mathrm{ClF}_{3} \mathrm{~N}_{5} \mathrm{O}_{2} \mathrm{~S}\right.$ requires 514.1291).

(S)-6-chloro-N-(1-(2-(3-chlorophenyl)hydrazineyl)-1-oxopropan-2-yl)-2-ethylimidazo[1,2-a]pyridine-3carboxamide, 36, following the standard procedure B, tert-butyl (S)-(1-(2-(3-chlorophenyl)hydrazineyl) -1-oxopropan-2-yl)carbamate $(0.1 \mathrm{~g}, 0.4 \mathrm{mmol})$ and 6-chloro-2-ethylimidazo[1,2-a]pyridine-3-carboxylic acid were transformed to afford the title compound as a white solid (28 mg, 17\%); m.p. 213-215 ${ }^{\circ} \mathrm{C}$; $v_{\max }$. (ATR) 3258, 2978, 1666, 1608, 1605, 1538, 1492, 1455, 1417, 1394, 1370, 1317, 1269, 1248, $1218,1159,1139,1110,1075,1044,993,950,906,850,802 \mathrm{~cm}^{-1} ; \delta_{\mathrm{H}}\left(400 \mathrm{MHz}, \mathrm{DMSO}-\mathrm{d}_{6}\right) 10.00$ $\left(1 \mathrm{H}, \mathrm{d}, J\right.$ 2 2, CONHNH), $9.07(1 \mathrm{H}, \mathrm{d}, J$ 2, $\mathrm{Ar}-\mathrm{H}), 8.31\left(1 \mathrm{H}, \mathrm{d}, J\right.$ 7, $\left.\mathrm{NHCH}\left(\mathrm{CH}_{3}\right) \mathrm{CO}\right), 8.13(1 \mathrm{H}, \mathrm{d}, J$ 2, CONHNH), $7.68(1 \mathrm{H}, \mathrm{d}, J$ 9, Ar-H), $7.48(1 \mathrm{H}, \mathrm{dd}, J$ 9, 2, Ar-H), $7.15(1 \mathrm{H}, \mathrm{t}, J$ 6 , Ar-H), $6.85(1 \mathrm{H}, \mathrm{s}$, $\mathrm{Ar}-\mathrm{H}), 6.74-6.70(2 \mathrm{H}, \mathrm{m}, \mathrm{Ar}-\mathrm{H}), 4.54\left(1 \mathrm{H}, \mathrm{q}, J\right.$ 7, $\left.\mathrm{NHCH}\left(\mathrm{CH}_{3}\right) \mathrm{CO}\right), 3.00\left(2 \mathrm{H}, \mathrm{q}, J 7, \mathrm{CH}_{2} \mathrm{CH}_{3}\right), 1.46(3 \mathrm{H}$, $\mathrm{d}, J$ 7, $\left.\mathrm{NHCH}\left(\mathrm{CH}_{3}\right) \mathrm{CO}\right), 1.28\left(3 \mathrm{H}, \mathrm{t}, J\right.$ 7, $\left.\mathrm{CH}_{2} \mathrm{CH}_{3}\right) ; \delta_{\mathrm{C}}\left(100 \mathrm{MHz}, \mathrm{DMSO}-\mathrm{d}_{6}\right)$ 172.8 (CONHNH), 161.1 (CONH), 151.9 (ipso-Ar-C), 151.3 (ipso-Ar-C), 143.7 (ipso-Ar-C), 134.1 (ipso-Ar-C), 130.7 (Ar-C), 127.6 (Ar-C), 125.3 (Ar-C), 120.1 (ipso-Ar-C), 118.3 (ipso-Ar-C), 117.7 (Ar-C), 116.3 (ipso-Ar-C), 112.0 (Ar-C), 111.4 (Ar-C), $48.3\left(\mathrm{NHCH}\left(\mathrm{CH}_{3}\right) \mathrm{CO}\right), 22.2\left(\mathrm{CH}_{2} \mathrm{CH}_{3}\right), 18.1\left(\mathrm{NHCH}\left(\mathrm{CH}_{3}\right) \mathrm{CO}\right), 13.4\left(\mathrm{CH}_{2} \mathrm{CH}_{3}\right) ; \mathrm{m} / \mathrm{z}\left(\mathrm{ES}^{+}\right)$ $420\left(\left[{ }^{35,35} \mathrm{Cl}\right] \mathrm{MH}^{+}\right), 422\left(\left[{ }^{35,37} \mathrm{Cl}\right] \mathrm{MH}^{+}\right), 424\left(\left[{ }^{37,37} \mathrm{Cl}\right] \mathrm{MH}^{+}\right)$; $\mathrm{HRMS}\left(\mathrm{ES}^{+}\right)$found $\left[{ }^{35,35} \mathrm{Cl}^{-\mathrm{MH}^{+}}, 420.0992\right.$ $\left(\mathrm{C}_{19} \mathrm{H}_{20}{ }^{35,35} \mathrm{Cl}_{2} \mathrm{~N}_{5} \mathrm{O}_{2}\right.$ requires 420.0994$)$.

(S)-6-chloro-N-(1-(2-(3-chlorophenyl)hydrazineyl)-4-(methylthio)-1-oxobutan-2-yl)-2-ethylimidazo[1,2 -a]pyridine-3-carboxamide, 37, following the standard procedure B, tert-Butyl N-[(1S)-1-[N'-(3-chlorophenyl) hydrazinecarbonyl]-3-(methylsulfanyl)propyl] carbamate $(70 \mathrm{mg}, \quad 0.23 \mathrm{mmol})$ and 6-chloro-2-ethylimidazo[1,2-a]pyridine-3-carboxylic acid were transformed to afford the title compound as a white solid (20 mg, 18\%); m.p. $188-191^{\circ} \mathrm{C}$; $v_{\max }$ (ATR) 3334, 2916, 1666, 1603, 1519, $1489,1417,1396,1341,1305,1288,1212,1151,1120,1048,998,963,872,847,814 \mathrm{~cm}^{-1} ; \delta_{\mathrm{H}}(400 \mathrm{MHz}$, DMSO-d $\left._{6}\right) 10.07(1 \mathrm{H}, \mathrm{d}, J$ 2, CONHNH), $9.04(1 \mathrm{H}, \mathrm{d}, J$ 2, Ar-H), $8.41(1 \mathrm{H}, \mathrm{d}, J$ 7, CONH), $8.15(1 \mathrm{H}$, $\mathrm{d}, J$ 2, CONHNH), $7.70(1 \mathrm{H}, \mathrm{d}, J$ 9, Ar-H), $7.48(1 \mathrm{H}, \mathrm{dd}, J$ 9, 2, Ar-H), $7.16(1 \mathrm{H}, \mathrm{t}, J$ 6, Ar-H), 6.86 $(1 \mathrm{H}, \mathrm{s}, \mathrm{Ar}-\mathrm{H}), 6.76-6.68(2 \mathrm{H}, \mathrm{m}, \mathrm{Ar}-\mathrm{H}), 4.59\left(1 \mathrm{H}, \mathrm{q}, J\right.$ 7, $\left.\mathrm{NHCH}\left(\mathrm{CH}_{2} \mathrm{CH}_{2} \mathrm{SCH}_{3}\right)\right), 3.00(2 \mathrm{H}, \mathrm{q}, J$, $\left.\mathrm{CH}_{2} \mathrm{CH}_{3}\right), 2.68-2.56\left(2 \mathrm{H}, \mathrm{m}, \mathrm{NHCH}\left(\mathrm{CH}_{2} \mathrm{CH}_{2} \mathrm{SCH}_{3}\right)\right), 2.12-2.06\left(5 \mathrm{H}, \mathrm{m}, \mathrm{NHCH}\left(\mathrm{CH}_{2} \mathrm{CH}_{2} \mathrm{SCH}_{3}\right), 1.28\right.$ 
$\left(3 \mathrm{H}, \mathrm{t}, \mathrm{J} 7, \mathrm{CH}_{2} \mathrm{CH}_{3}\right) ; \delta_{\mathrm{C}}\left(176 \mathrm{MHz}, \mathrm{DMSO}-\mathrm{d}_{6}\right) 172.0(\mathrm{CONHNH}), 161.6(\mathrm{CONH}), 152.0$ (ipso-Ar-C), 151.3 (ipso-Ar-C), 143.8 (ipso-Ar-C), 134.1 (ipso-Ar-C), 130.7 (Ar-C), 127.6 (Ar-C), 125.3 (Ar-C), 120.1 (ipso-Ar-C), 118.3 (ipso-Ar-C), 117.7 (Ar-C), 116.4 (ipso-Ar-C), 112.0 (Ar-C), 111.4 (Ar-C), 52.0 $\left(\mathrm{NHCH}\left(\mathrm{CH}_{2} \mathrm{CH}_{2} \mathrm{SCH}_{3}\right)\right), 31.2\left(\mathrm{NHCH}\left(\mathrm{CH}_{2} \mathrm{CH}_{2} \mathrm{SCH}_{3}\right)\right), 31.1\left(\mathrm{NHCH}\left(\mathrm{CH}_{2} \mathrm{CH}_{2} \mathrm{SCH}_{3}\right)\right), 22.3\left(\mathrm{CH}_{2} \mathrm{CH}_{3}\right)$, $15.1\left(\mathrm{NHCH}\left(\mathrm{CH}_{2} \mathrm{CH}_{2} \mathrm{SCH}_{3}\right)\right), 13.6\left(\mathrm{CH}_{2} \mathrm{CH}_{3}\right) ; m / z\left(\mathrm{ES}^{+}\right) 480\left(\left[{ }^{35,35} \mathrm{Cl} \mathrm{MH}^{+}\right), 482\left(\left[{ }^{35,37} \mathrm{Cl}\right] \mathrm{MH}^{+}\right), 484\right.$ $\left(\left[{ }^{37,37} \mathrm{Cl}\right] \mathrm{MH}^{+}\right)$; HRMS $\left(\mathrm{ES}^{+}\right)$found $\left[{ }^{35,35} \mathrm{Cl}\right] \mathrm{MH}^{+}, 480.1026\left(\mathrm{C}_{21} \mathrm{H}_{24}{ }^{35,35} \mathrm{Cl}_{2} \mathrm{~N}_{5} \mathrm{O}_{2} \mathrm{~S}\right.$ requires 480.1028$)$.

\subsection{Biological Assessment}

\subsubsection{Cell Lines}

The bacterial species and cell lines used in this study can be found in Table 2.

Table 2. Results of synthesis of the substituted amino acid hydrazides and the antibacterial activity and cytotoxicity.

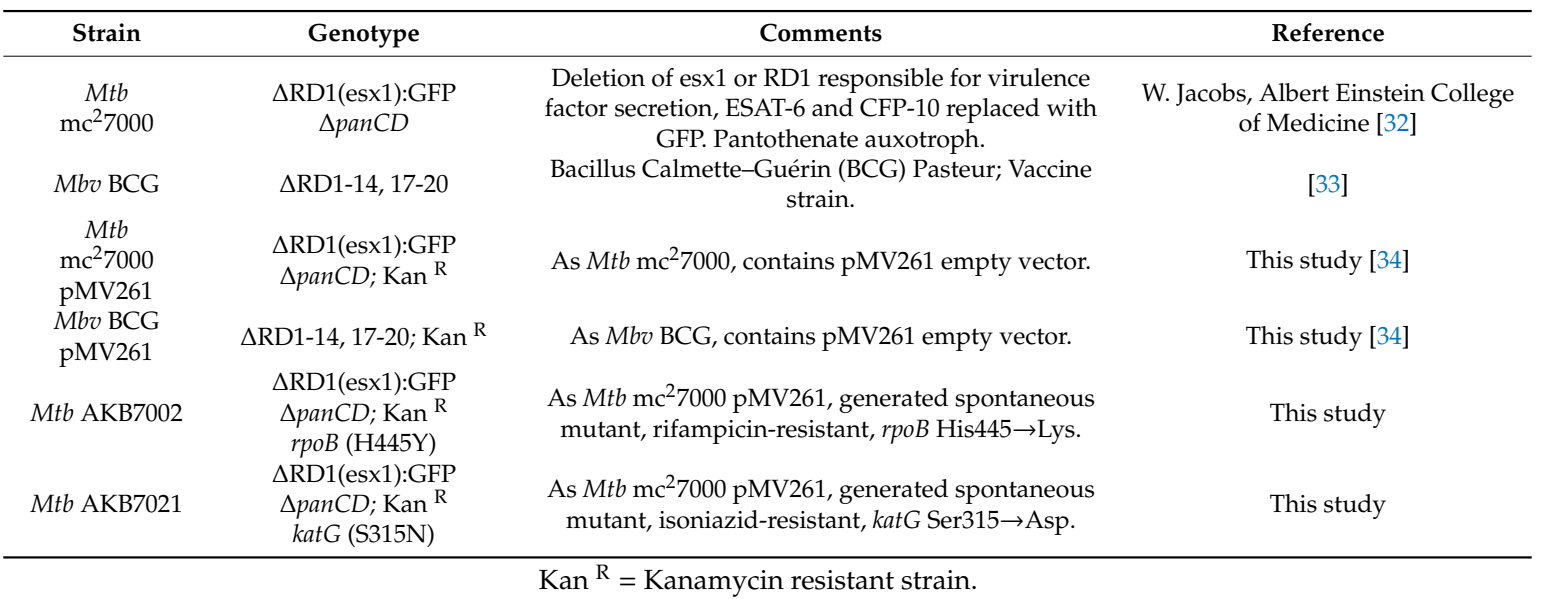

The cell line utilized for cytotoxicity studies were the murine macrophage cell line RAW264.7 (ATCC TIB-71).

\subsubsection{Bacterial Growth Conditions}

Mycobacterial species were cultured in either Middlebrook 7H9 broth or Middlebrook 7H10 agar media supplemented with albumin-dextrose-catalase (ADC) or oleic acid-albumin-dextrose-catalase (OADC) enrichments and $0.2 \%$ glycerol, $0.2 \%$ casamino acids, $24 \mu \mathrm{g} / \mathrm{mL}$ pantothenate, $1 \mu \mathrm{g} / \mathrm{mL}$ penicillin G, $10 \mu \mathrm{g} / \mathrm{mL}$ cyclohexamide, purchased from BD Biosciences. All reagents were purchased from Sigma-Aldrich, Gillingham, UK unless stated otherwise.

\subsubsection{Spontaneous Mutant Generation}

$\mathrm{Mtb} \mathrm{mc}^{2} 7000$ mono-resistant mutants were generated by plating $1 \times 10^{8}$ mid-log cells on solid media (7H11 agar supplemented with $10 \%$ OADC and $0.2 \%$ glycerol, $0.2 \%$ casamino acids, $24 \mu \mathrm{g} / \mathrm{mL}$ pantothenate, $1 \mu \mathrm{g} / \mathrm{mL}$ penicillin $\mathrm{G}, 10 \mu \mathrm{g} / \mathrm{mL}$ cyclohexamide) containing $0.1,0.2,0.4,1.6$ and $3.2 \mu \mathrm{g} / \mathrm{mL}$ of RIF or INH. Plates were incubated at $37{ }^{\circ} \mathrm{C}, 5 \% \mathrm{CO}_{2}$ until the growth of colonies was visible. Spontaneous resistant mutants were confirmed by minimum inhibitory concentration (MIC) testing in liquid media as described below (REMA). The genomic DNA from validated mutants was extracted, and Next Generation Sequencing (NGS) analysis was performed to confirm mutation locations.

\subsubsection{Bacterial Growth Inhibition Assays}

Bacterial minimum inhibition concentrations (MIC) were determined using the resazurin microtiter assay (REMA) method, according to Palomino et al. [35]. Stock solutions of the tested compounds were 
prepared in sterile dimethyl sulfoxide (DMSO), then diluted in Middlebrook 7H9 broth (Difco, Detroit, MI, USA) supplemented with oleic acid, albumin, dextrose and catalase (OADC enrichment) to obtain a final compound concentration range of $0.0625-64 \mu \mathrm{g} / \mathrm{mL}$. A suspension of the test Mycobacterium was cultured in Middlebrook 7H9 broth supplemented with 10\% OADC and $0.2 \%$ glycerol, $0.2 \%$ casamino acids, $24 \mu \mathrm{g} / \mathrm{mL}$ pantothenate, $1 \mu \mathrm{g} / \mathrm{mL}$ penicillin G, $10 \mu \mathrm{g} / \mathrm{mL}$ cyclohexamide and $0.05 \%$ Tween 80 for one week at $37{ }^{\circ} \mathrm{C}$ in an atmosphere of $5 \% \mathrm{CO}_{2}$. The concentration was adjusted at McFarland 0.5 and diluted to $1 \times 10^{6} \mathrm{CFU} / \mathrm{mL}$ and diluted in growth media 1:25. A $100 \mu \mathrm{L}$ of the inoculum was added to each well of a 96-well microplate together with $100 \mu \mathrm{L}$ of the compounds. The plate was incubated at $37^{\circ} \mathrm{C}$ in an atmosphere of $5 \% \mathrm{CO}_{2}$. After 5 days, $10 \mu \mathrm{L} 0.2 \%(w / v)$ resazurin (solubilized in sterile water) was added. MIC $_{99}$ was defined as the lowest concentration resulting in $99 \%$ inhibition of growth of Mycobacterium resulting in no colour change. Samples were set up in quadruplet and tested in two independent assays.

\subsubsection{Determination of Cytotoxicity of Compounds against Mammalian Macrophages}

RAW 264.7 cell lines were purchased from American Type Culture Collection, Middlesex, UK (ATCC1 TIB-71 ${ }^{\mathrm{TM}}$ ). Cells were cultured in DMEM high glucose medium, supplemented with 10\% FBS, $2 \mathrm{mM}$ L-Glutamine and $1 \%$ penicillin/streptomycin, at $37^{\circ} \mathrm{C}$ in a humidified incubator containing $5 \%$ $\mathrm{CO}_{2}$. Cells were passaged after reaching $90 \%$ confluence.

Cells, in logarithmic growth, were plated in 96-well plates at a density of $1 \times 10^{4}$ cells/well in $200 \mu \mathrm{L}$ of media and incubated for $24 \mathrm{~h}$. The media on the cells was removed and replaced with $180 \mu \mathrm{L}$ of fresh media. To each respective well, $20 \mu \mathrm{L}$ of test compound at a concentration of $1 \mathrm{mM}$ was added, resulting in a final compound concentration of $100 \mu \mathrm{M}$. To control wells, DMSO drug solvent was added. In all wells, the final concentration of DMSO did not exceed $0.1 \%$. Following incubation for a further $96 \mathrm{~h}$ at $37{ }^{\circ} \mathrm{C}$ in a humidified incubator containing $5 \%$ $\mathrm{CO}_{2}$, all media was removed and replaced with $100 \mu \mathrm{L}$ of fresh media. To each well, $20 \mu \mathrm{L}$ of 3-(4,5-dimethylthiazol-2-yl)-5-(3-carboxymethoxyphenyl)-2-(4-sulfophenyl)-2H-tetrazolium, inner salt (MTS) assay solution (Promega, Madison, WI, USA) was added, and the plate was incubated in the dark at $37^{\circ} \mathrm{C}$ for $4 \mathrm{~h}$. Finally, the absorbance of each well was recorded at $490 \mathrm{~nm}$ using a microplate reader. The percentage of cell viability was calculated relevant to the compound solvent (DMSO) control.

\section{Conclusions}

With the end TB strategy faltering and the pipeline for new TB drugs being minimal, the requirement for novel architectures to combat the rise in drug-resistant TB infections is of paramount importance. The existing benzoxa-[2,1,3]-diazole amino acid hydrazide scaffolds were modified in order to demonstrate that the removal of the benzoxa-[2,1,3]-diazole moiety and replacement, utilising a scaffold hopping approach with relevant pharmacophores from existing anti-tubercular drugs, was a feasible tactic. The use of known chemical moieties present in molecules with anti-TB activity, such as quinoline or imidazo [1,2-a]pyridine-3-carboxy substituents, have provided a variety of antibacterial activities similar or greater to that with the benzoxa-[2,1,3]-diazole moiety and molecules with defined selectivity against drug-resistant strains of $M t b$ (Table 1 and [16]). In addition, the greater antibacterial activity observed between 3-chlorophenyl-substituted hydrazides compared to the 4-trifluorophenyl-substituted hydrazides remains to be elucidated; however, this may indicate a preferential binding pocket in the target site, favouring meta-substituted aromatic rings.

Overall, $N$-substituted amino acid hydrazides have provided a versatile scaffold for elaboration with known pharmacophores from existing drugs to provide active and clinically translatable anti-tubercular drugs. Whilst the exact mechanism of action is not understood, phenotypic observations have shown that these compounds favour isoniazid-resistant $M t b$ strains and so may target a pathway upstream of the site of INH activity or one involving perturbation of the ETC within mycobacteria. This hypothesis is yet to be tested and is currently under investigation. 
Supplementary Materials: The following are available online at http://www.mdpi.com/1420-3049/25/10/2387/ s1, Figure S1: Representative comparison data between our current compounds (this study) and our lead benzoxa-[2,1,3]-diazoles compounds from our previous study, Figure S2: ${ }^{1} \mathrm{H}$ and ${ }^{13} \mathrm{C}$ NMR for all new compounds produced in this study.

Author Contributions: Conceptualization, J.D.S.; Data curation, A.K.B., A.K.B.A., F.M.A.A., J.L.B., J.H.G., Y.L. and J.D.S.; Formal analysis, A.K.B., J.H.G. and J.D.S.; Investigation, A.K.B., A.K.B.A., F.M.A.A., J.L.B., J.H.G., Y.L. and J.D.S.; Methodology, A.K.B., J.H.G. and J.D.S.; Project administration, J.D.S.; Resources, A.K.B., J.H.G. and J.D.S.; Supervision, A.K.B., J.H.G. and J.D.S.; Validation, A.K.B., J.H.G. and J.D.S.; Visualization, A.K.B., J.H.G. and J.D.S.; Writing-original draft, J.D.S.; Writing-review and editing, A.K.B., J.H.G. and J.D.S. All authors have read and agreed to the published version of the manuscript.

Funding: This research received no external funding.

Acknowledgments: We would also like to thank Durham University and Newcastle University for financial support of this work (J.L.B. MChem project), Alan M. Kenwright (University of Durham) and Corinne Wills for assistance with NMR spectroscopy and Jackie A. Mosely (University of Teeside) for mass spectra.

Conflicts of Interest: The authors declare no conflict of interest.

\section{References}

1. World Health Organisation. Global Tuberculosis Report; World Health Organisation: Geneva, Switzerland, 2019.

2. WHO End TB Strategy. Available online: https://www.who.int/tb/post2015_strategy/en/ (accessed on 10 September 2019).

3. Denny, W.A.; Palmer, B.D. The nitroimidazooxazines (PA-824 and analogS): Structure-activity relationship and mechanistic studies. Future Med. Chem. 2010, 2, 1295-1304. [CrossRef]

4. Christophe, T.; Jackson, M.; Jeon, H.K.; Fenistein, D.; Contreras-Dominguez, M.; Kim, J.; Genovesio, A.; Carralot, J.P.; Ewann, F.; Kim, E.H.; et al. High content screening identifies decaprenyl-phosphoribose 2' epimerase as a target for intracellular antimycobacterial inhibitors. PLoS Pathog. 2009, 5, e1000645. [CrossRef]

5. Zhang, Y.; Shi, W.; Zhang, W.; Mitchison, D. Mechanisms of Pyrazinamide Action and Resistance. Microbiol. Spectr. 2013, 2, 1-12.

6. Bloemberg, G.V.; Keller, P.M.; Stucki, D.; Trauner, A.; Borrell, S.; Latshang, T.; Coscolla, M.; Rothe, T.; Homke, R.; Ritter, C.; et al. Acquired Resistance to Bedaquiline and Delamanid in Therapy for Tuberculosis. N. Engl. J. Med. 2015, 373, 1986-1988. [CrossRef]

7. Liu, Y.; Matsumoto, M.; Ishida, H.; Ohguro, K.; Yoshitake, M.; Gupta, R.; Geiter, L.; Hafkin, J. Delamanid: From discovery to its use for pulmonary multidrug-resistant tuberculosis (MDR-TB). Tuberculosis 2018, 111, 20-30. [CrossRef]

8. Schena, E.; Nedialkova, L.; Borroni, E.; Battaglia, S.; Cabibbe, A.M.; Niemann, S.; Utpatel, C.; Merker, M.; Trovato, A.; Hofmann-Thiel, S.; et al. Delamanid susceptibility testing of Mycobacterium tuberculosis using the resazurin microtitre assay and the BACTEC MGIT 960 system. J. Antimicrob. Chemother. 2016, 71, 1532-1539. [CrossRef]

9. Fujiwara, M.; Kawasaki, M.; Hariguchi, N.; Liu, Y.; Matsumoto, M. Mechanisms of resistance to delamanid, a drug for Mycobacterium tuberculosis. Tuberculosis 2018, 108, 186-194. [CrossRef]

10. Bald, D.; Villellas, C.; Lu, P.; Koul, A. Targeting Energy Metabolism in Mycobacterium tuberculosis, a New Paradigm in Antimycobacterial Drug Discovery. MBio 2017, 8, e0272-17. [CrossRef]

11. Koul, A.; Dendouga, N.; Vergauwen, K.; Molenberghs, B.; Vranckx, L.; Willebrords, R.; Ristic, Z.; Lill, H.; Dorange, I.; Guillemont, J.; et al. Diarylquinolines target subunit c of mycobacterial ATP synthase. Nat. Chem. Biol. 2007, 3, 323-324. [CrossRef]

12. Andries, K.; Verhasselt, P.; Guillemont, J.; Gohlmann, H.W.; Neefs, J.M.; Winkler, H.; Van Gestel, J.; Timmerman, P.; Zhu, M.; Lee, E.; et al. A diarylquinoline drug active on the ATP synthase of Mycobacterium tuberculosis. Science 2005, 307, 223-227. [CrossRef]

13. Tahlan, K.; Wilson, R.; Kastrinsky, D.B.; Arora, K.; Nair, V.; Fischer, E.; Barnes, S.W.; Walker, J.R.; Alland, D.; Barry, C.E.; et al. SQ109 targets MmpL3, a membrane transporter of trehalose monomycolate involved in mycolic acid donation to the cell wall core of Mycobacterium tuberculosis. Antimicrob. Agents Chemother. 2012, 56, 1797-1809. [CrossRef] 
14. De Jager, V.R.; Dawson, R.; van Niekerk, C.; Hutchings, J.; Kim, J.; Vanker, N.; van der Merwe, L.; Choi, J.; Nam, K.; Diacon, A.H. Telacebec (Q203), a New Antituberculosis Agent. N. Engl. J. Med. 2020, 382, 1280-1281. [CrossRef]

15. Yano, T.; Kassovska-Bratinova, S.; Teh, J.S.; Winkler, J.; Sullivan, K.; Isaacs, A.; Schechter, N.M.; Rubin, H. Reduction of clofazimine by mycobacterial type $2 \mathrm{NADH}$ :quinone oxidoreductase: A pathway for the generation of bactericidal levels of reactive oxygen species. J. Biol. Chem. 2011, 286, 10276-10287. [CrossRef]

16. Brown, A.K.; Aljohani, A.K.B.; Gill, J.H.; Steel, P.G.; Sellars, J.D. Identification of Novel Benzoxa-[2,1,3]-diazole Substituted Amino Acid Hydrazides as Potential Anti-Tubercular Agents. Molecules 2019, 24, 811. [CrossRef]

17. Surase, Y.B.; Samby, K.; Amale, S.R.; Sood, R.; Purnapatre, K.P.; Pareek, P.K.; Das, B.; Nanda, K.; Kumar, S.; Verma, A.K. Identification and synthesis of novel inhibitors of mycobacterium ATP synthase. Bioorg. Med. Chem. Lett. 2017, 27, 3454-3459. [CrossRef]

18. Wavhale, R.D.; Martis, E.A.F.; Ambre, P.K.; Wan, B.; Franzblau, S.G.; Iyer, K.R.; Raikuvar, K.; Macegoniuk, K.; Berlicki, L.; Nandan, S.R.; et al. Discovery of new leads against Mycobacterium tuberculosis using scaffold hopping and shape based similarity. Bioorg. Med. Chem. 2017, 25, 4835-4844. [CrossRef]

19. Lone, M.Y.; Athar, M.; Gupta, V.K.; Jha, P.C. Identification of Mycobacterium tuberculosis enoyl-acyl carrier protein reductase inhibitors: A combined in-silico and in-vitro analysis. J. Mol. Graph. Model. 2017, 76, 172-180. [CrossRef]

20. Igarashi, M.; Ishizaki, Y.; Takahashi, Y. New antituberculous drugs derived from natural products: Current perspectives and issues in antituberculous drug development. J. Antibiot. 2018, 71, 15-27. [CrossRef]

21. Scalacci, N.; Brown, A.K.; Pavan, F.R.; Ribeiro, C.M.; Manetti, F.; Bhakta, S.; Maitra, A.; Smith, D.L.; Petricci, E.; Castagnolo, D. Synthesis and SAR evaluation of novel thioridazine derivatives active against drug-resistant tuberculosis. Eur. J. Med. Chem. 2017, 127, 147-158. [CrossRef]

22. Vilcheze, C.; Jacobs, W.R., Jr. Resistance to Isoniazid and Ethionamide in Mycobacterium tuberculosis: Genes, Mutations, and Causalities. Microbiol. Spectr. 2014, 2, 431-453. [CrossRef]

23. Gonec, T.; Pospisilova, S.; Kauerova, T.; Kos, J.; Dohanosova, J.; Oravec, M.; Kollar, P.; Coffey, A.; Liptaj, T.; Cizek, A.; et al. N-Alkoxyphenylhydroxynaphthalenecarboxamides and Their Antimycobacterial Activity. Molecules 2016, 21, 1068. [CrossRef]

24. Zeng, S.; Soetaert, K.; Ravon, F.; Vandeput, M.; Bald, D.; Kauffmann, J.M.; Mathys, V.; Wattiez, R.; Fontaine, V. Isoniazid Bactericidal Activity Involves Electron Transport Chain Perturbation. Antimicrob. Agents Chemother. 2019, 63, e01841-18. [CrossRef]

25. Baptista, R.; Fazakerley, D.M.; Beckmann, M.; Baillie, L.; Mur, L.A.J. Untargeted metabolomics reveals a new mode of action of pretomanid (PA-824). Sci. Rep. 2018, 8, 5084-5091. [CrossRef]

26. Edwards, D.I. Nitroimidazole drugs-action and resistance mechanisms I. Mechanism of action. J. Antimicrob. Chemother. 1993, 31, 9-20. [CrossRef]

27. Samala, G.; Nallangi, R.; Devi, P.B.; Saxena, S.; Yadav, R.; Sridevi, J.P.; Yogeeswari, P.; Sriram, D. Identification and development of 2-methylimidazo[1,2-a]pyridine-3-carboxamides as Mycobacterium tuberculosis pantothenate synthetase inhibitors. Bioorg. Med. Chem. 2014, 22, 4223-4232. [CrossRef]

28. Wang, A.; Wang, H.; Geng, Y.; Fu, L.; Gu, J.; Wang, B.; Lv, K.; Liu, M.; Tao, Z.; Ma, C.; et al. Design, synthesis and antimycobacterial activity of less lipophilic Q203 derivatives containing alkaline fused ring moieties. Bioorg. Med. Chem. 2019, 27, 813-821. [CrossRef]

29. Wang, H.; Wang, A.; Gu, J.; Fu, L.; Lv, K.; Ma, C.; Tao, Z.; Wang, B.; Liu, M.; Guo, H.; et al. Synthesis and antitubercular evaluation of reduced lipophilic imidazo[1,2-a]pyridine-3-carboxamide derivatives. Eur. J. Med. Chem. 2019, 165, 11-17. [CrossRef]

30. Pethe, K.; Bifani, P.; Jang, J.; Kang, S.; Park, S.; Ahn, S.; Jiricek, J.; Jung, J.; Jeon, H.K.; Cechetto, J.; et al. Discovery of Q203, a potent clinical candidate for the treatment of tuberculosis. Nat. Med. 2013, 19, 1157-1160. [CrossRef]

31. Vilcheze, C.; Weisbrod, T.R.; Chen, B.; Kremer, L.; Hazbon, M.H.; Wang, F.; Alland, D.; Sacchettini, J.C.; Jacobs, W.R., Jr. Altered NADH/NAD+ ratio mediates coresistance to isoniazid and ethionamide in mycobacteria. Antimicrob. Agents Chemother. 2005, 49, 708-720. [CrossRef]

32. Sambandamurthy, V.K.; Derrick, S.C.; Hsu, T.; Chen, B.; Larsen, M.H.; Jalapathy, K.V.; Chen, M.; Kim, J.; Porcelli, S.A.; Chan, J.; et al. Mycobacterium tuberculosis DeltaRD1 DeltapanCD: A safe and limited replicating mutant strain that protects immunocompetent and immunocompromised mice against experimental tuberculosis. Vaccine 2006, 24, 6309-6320. [CrossRef] 
33. Brosch, R.; Gordon, S.V.; Garnier, T.; Eiglmeier, K.; Frigui, W.; Valenti, P.; Dos Santos, S.; Duthoy, S.; Lacroix, C.; Garcia-Pelayo, C.; et al. Genome plasticity of BCG and impact on vaccine efficacy. Proc. Natl. Acad. Sci. USA 2007, 104, 5596-5601. [CrossRef] [PubMed]

34. Stover, C.K.; de la Cruz, V.F.; Fuerst, T.R.; Burlein, J.E.; Benson, L.A.; Bennett, L.T.; Bansal, G.P.; Young, J.F.; Lee, M.H.; Hatfull, G.F.; et al. New use of BCG for recombinant vaccines. Nature 1991, 351, 456-460. [CrossRef] [PubMed]

35. Palomino, J.C.; Martin, A.; Camacho, M.; Guerra, H.; Swings, J.; Portaels, F. Resazurin microtiter assay plate: Simple and inexpensive method for detection of drug resistance in Mycobacterium tuberculosis. Antimicrob. Agents Chemother. 2002, 46, 2720-2722. [CrossRef] [PubMed]

Sample Availability: Samples of all the compounds are available from the authors.

(C) 2020 by the authors. Licensee MDPI, Basel, Switzerland. This article is an open access article distributed under the terms and conditions of the Creative Commons Attribution (CC BY) license (http://creativecommons.org/licenses/by/4.0/). 